\title{
ON JACOBIAN GROUP ARITHMETIC FOR TYPICAL DIVISORS ON CURVES
}

\author{
KAMAL KHURI-MAKDISI
}

\begin{abstract}
In a previous joint article with F. Abu Salem, we gave efficient algorithms for Jacobian group arithmetic of "typical" divisor classes on $C_{3,4}$ curves, improving on similar results by other authors. At that time, we could only state that a general divisor was typical, and hence unlikely to be encountered if one implemented these algorithms over a very large finite field. This article pins down an explicit characterization of these typical divisors, for an arbitrary smooth projective curve of genus $g \geq 1$ having at least one rational point. We give general algorithms for Jacobian group arithmetic with these typical divisors, and prove not only that the algorithms are correct if various divisors are typical, but also that the success of our algorithms provides a guarantee that the resulting output is correct and that the resulting input and/or output divisors are also typical. These results apply in particular to our earlier algorithms for $C_{3,4}$ curves. As a byproduct, we obtain a further speedup of approximately $15 \%$ on our previous algorithms for $C_{3,4}$ curves.
\end{abstract}

\section{INTRODUCTION}

Let $C$ be a smooth projective algebraic curve of genus $g \geq 1$ over a field $\mathbb{K}$, and assume that $C$ has a $\mathbb{K}$-rational point $P_{\infty}$. Let $\mathcal{R}$ be the coordinate ring of the affine curve $C-\left\{P_{\infty}\right\}$; then the group of $\mathbb{K}$-rational points of the Jacobian of $C$ can be identified with the ideal class group of the Dedekind domain $\mathcal{R}$. A previous series of articles (by three different groups of authors) on certain degree 3 covers of $\mathbf{P}^{1}$, particularly $C_{3,4}$ curves BEFG04, BEFG05, FO04, FOR08, ASKM07, OT13, gives explicit formulas for group arithmetic in the Jacobian of $C$ when $\mathbb{K}$ is a large finite field, under a certain genericity assumption on the divisors whose classes are being added in the Jacobian. This genericity assumption was first introduced in BEFG05, where such divisors were called "typical"; all the articles above give fast algorithms for Jacobian group arithmetic under the hypothesis that the divisors (also, in fact, the pairs of divisors) one encounters are typical, and that the result of the group operation is typical. The above articles, however, do not include a test to verify whether the input divisors or output data are in fact typical, so that in principle the algorithms might return wrong results without this being detected during the computation. It is desirable to know when such algorithms have failed, so that one can redo the computation using slightly slower algorithms that work for all divisors (for example, those in KM04, KM07]).

In this article, we give a straightforward explicit condition for a divisor to be typical, for arbitrary $C$. For the $C_{3,4}$ case, we show that the algorithms in ASKM07, which involve two inversions (and approximately 125 multiplications) in $\mathbb{K}$ per group

2000 Mathematics Subject Classification. 14Q05, 11Y16, 14H40, 11G20.

November 14, 2017. 
operation in the Jacobian of a $C_{3,4}$ curve, give correct results and yield typical divisors as output, provided that both inversions can be carried out, i.e., that one encounters nonzero elements of $\mathbb{K}$ at those two moments. Our general criterion for typicality can be expressed in terms of the rank of certain matrices, or equivalently in terms of the structure of suitable Gröbner bases for the ideal $I_{D}$ and its first syzygies. For an arbitrary curve $C$ with distinguished point $P_{\infty}$, we describe a modification of the algorithms from [ASKM07, that allows us to carry out Jacobian arithmetic for typical elements. To our knowledge, this is the first set of algorithms for typical divisors on curves that (1) works with a precise definition of typicality (and a weaker notion of semi-typicality), (2) gives necessary and sufficient conditions on the typicality of the input and/or output divisors to guarantee success of the algorithms, and (3) can certify that the end result of one's operations is correct upon success, or else identify non-typical divisors encountered in the computation. Although some of the proofs are delicate, we find the way in which the different ranks of subspaces fit together both intricate and pleasing.

As a result of our current investigations, we discovered along the way a nontrivial speedup of the algorithms of [ASKM07, that saves 19 multiplications in $\mathbb{K}$ per operation in the Jacobian, a speedup of approximately $15 \%$. This is described in the appendix to this article.

\section{TYPICAL DIVISORS}

All divisors that we consider in this article are $\mathbb{K}$-rational. The reader is however encouraged to replace $\mathbb{K}$ by its algebraic closure $\overline{\mathbb{K}}$, so that every divisor is a sum of geometric points, without worrying about rationality. This does not affect our results, since everything we do boils down to the interplay between different $\mathbb{K}$ rational subspaces of various vector spaces, which in turn can be recast in terms of the ranks of various matrices with entries in $\mathbb{K}$; these ranks are unaffected by extension of scalars.

Definition 2.1. (1) Let $\mathcal{R}=\mathbb{K}\left[C-\left\{P_{\infty}\right\}\right]$ be the affine coordinate ring of $C$, as in the introduction, and let $N \geq 0$. For $f \in \mathcal{R}$, we define its degree to be the order of its pole at $P_{\infty}$ :

$$
\operatorname{deg} f=-v_{P_{\infty}}(f) ; \quad \text { by convention, let } \operatorname{deg} 0=-\infty .
$$

(2) We define the basic Riemann-Roch space $W^{N}$ by

$$
W^{N}=H^{0}\left(C, \mathcal{O}_{C}\left(N P_{\infty}\right)\right)=\{f \in \mathcal{R} \mid \operatorname{deg}(f) \leq N\},
$$

consisting of elements $f$ of the function field $\mathbb{K}(C)$ that are regular everywhere except for a pole of order at most $N$ at $P_{\infty}$. Thus $\mathcal{R}=\bigcup_{N \geq 0} W^{N}$.

(3) We define a good divisor $D$ on $C$ to be an effective ( $\mathbb{K}$-rational) divisor disjoint from $P_{\infty}$.

(4) For $f \in \mathcal{R}$, we will generally write $\operatorname{div} f$ to refer to the affine part of the divisor, unless otherwise specified. This means that $\operatorname{div} f$ will completely ignore the component at $P_{\infty}$, and will hence be a good divisor; we then have $\operatorname{deg}(\operatorname{div} f)=\operatorname{deg} f$. The actual "full" divisor of $f$ is the degree zero divisor $\operatorname{div} f-(\operatorname{deg} f) P_{\infty}$.

(5) For a good divisor $D$, we define the $\mathbb{K}$-rational subspace $W_{D}^{N} \subset W^{N}$ by

$$
W_{D}^{N}=H^{0}\left(C, \mathcal{O}_{C}\left(N P_{\infty}-D\right)\right)=W^{N} \cap I_{D},
$$


where $I_{D} \subset \mathcal{R}$ is the ideal of functions vanishing on $D$ :

$$
I_{D}=\{f \in \mathcal{R} \mid \operatorname{div} f \geq D\} .
$$

Hence $W_{D}^{N}$ is the space of elements of $I_{D}$ of degree at most $N$. We have $\operatorname{dim}_{\mathbb{K}} \mathcal{R} / I_{D}=\operatorname{deg} D$.

By Riemann-Roch, we have the following dimensions:

$$
\begin{aligned}
& \operatorname{dim} W^{N}=N+1-g, \text { for } N \geq 2 g-1, \\
& \operatorname{dim} W_{D}^{N}=N-\operatorname{deg} D+1-g, \text { for } N \geq \operatorname{deg} D+2 g-1 .
\end{aligned}
$$

It is moreover known that for "most" good divisors $D$ with $d \geq g$, the formula for $\operatorname{dim} W_{D}^{N}$ above is actually valid in the larger range $N \geq \operatorname{deg} D+g-1$. We do not use this fact yet, and will come back to it later.

We also recall the notion of a base point free line bundle $\mathcal{L}$. This means that the sections of $H^{0}(C, \mathcal{L})$ do not vanish simultaneously on any (geometric) point in $C(\overline{\mathbb{K}})$, or equivalently on any nontrivial $\mathbb{K}$-rational effective divisor $E$. In the setting where $\mathcal{L}=\mathcal{O}_{C}\left(N P_{\infty}-D\right)$, this means that the only common zeros of the elements $f \in W_{D}^{N}$ occur on $D$ - equivalently, the elements of $W_{D}^{N}$ generate the ideal $I_{D}$ - and that moreover there exists a nonzero $f \in W_{D}^{N}$ with $\operatorname{deg} f=N$, to ensure that no excess "vanishing" occurs at $P_{\infty}$. The following result is a standard consequence of Riemann-Roch:

If $\operatorname{deg} \mathcal{L} \geq 2 g$, then $\mathcal{L}$ is base point free.

Once again, it is known that "most" line bundles of degree $\operatorname{deg} \mathcal{L} \geq g+1$ are base point free, and we will come back to this point later in this section. We will frequently abuse terminology and say " $W_{D}^{N}$ is base point free" when we really mean the line bundle $\mathcal{O}_{C}\left(N P_{\infty}-D\right)$.

The following two definitions, of typical and semi-typical divisors, are fundamental to our work. Our immediate aim is to show that typical divisors are semi-typical (Proposition 2.3 below), and that these notions depend only on the linear equivalence class of the divisor (Corollary 2.6 below, which actually proves something more). Once those results are established, we show that all but a "small" set of divisors is typical (Proposition 2.9 below), and mention numerical bounds (proved in [KM16]) for the probability that, over a finite field, a random divisor is typical or semi-typical (Theorem 2.10).

Definition 2.2. In the definitions below, recall that $g \geq 1$, so that $d \geq g \geq 1$.

(1) A divisor $D$ is called typical if (i) $D$ is a good divisor of degree $d \geq g$, and (ii) there exist $s \in W_{D}^{d+g}$ and $t \in W_{D}^{d+g+1}$ with $s W^{2 g}+t W^{2 g-1}+W^{d+g-1}=$ $W^{d+3 g}$.

(2) A divisor $D$ is called semi-typical if (i) $D$ is a good divisor of degree $d \geq g$, and (ii) $W_{D}^{d+g-1}=0$.

Proposition 2.3. Let $D$ be a typical divisor as above. Then:

(1) The sum in the definition is in fact a direct sum $s W^{2 g} \oplus t W^{2 g-1} \oplus W^{d+g-1}=$ $W^{d+3 g}$, and we also have a direct sum $s W^{2 g} \oplus t W^{2 g-1}=W_{D}^{d+3 g}$.

(2) The divisor $D$ is semi-typical.

(3) The pair $\{s, t\}$ is an IGS (i.e., ideal generating set) for $D$, in the terminology of [KM07]. This means in our context that the locus of common 
zeros of $s$ and $t$ is precisely $D$; hence $s, t$ generate the ideal $I_{D} \subset \mathcal{R}$. In particular, $W_{D}^{d+g+1}$, which has degree $g+1$, is base point free.

(4) We have that $\operatorname{deg} s=d+g$, $\operatorname{deg} t=d+g+1$, and $W_{D}^{d+g+1}$ is 2-dimensional, with basis $\{s, t\}$; any other choice of $s^{\prime}, t^{\prime} \in W_{D}^{d+g+1}$ with the same degrees is also a basis of that space, and satisfies $s^{\prime} W^{2 g}+t^{\prime} W^{2 g-1}+W^{d+g-1}=W^{d+3 g}$.

Proof. The fact that the first sum is direct follows by counting dimensions in the equality $s W^{2 g}+t W^{2 g-1}+W^{d+g-1}=W^{d+3 g}$. For example, once $s \neq 0$, we have $\operatorname{dim} s W^{2 g}=\operatorname{dim} W^{2 g}=g+1$, and similarly $\operatorname{dim} t W^{2 g-1}=g, \operatorname{dim} W^{d+g-1}=d$, and $\operatorname{dim} W^{d+3 g}=d+2 g+1$. This implies in particular that $s, t \neq 0$, and in fact that $s$ and $t$ are $\mathbb{K}$-linearly independent, since $s \in s W^{2 g}$ and $t \in t W^{2 g-1}$. By counting dimensions again, the inclusion $s W^{2 g}+t W^{2 g-1}\left(=s W^{2 g} \oplus t W^{2 g-1}\right) \subset$ $W_{D}^{d+3 g}$ must be an equality. Now directness of the first sum implies that the intersection $W^{d+g-1} \cap\left(s W^{2 g}+t W^{2 g-1}\right)$ must be zero, which can be rewritten as $0=W^{d+g-1} \cap W_{D}^{d+3 g}=W_{D}^{d+g-1}$, so $D$ is semi-typical.

Since $W_{D}^{d+3 g}$ is base point free, we deduce from $s W^{2 g}+t W^{2 g-1}=W_{D}^{d+3 g}$ that $\{s, t\}$ is an IGS for $D$. Also, $s$ and $t$ are linearly independent, so $\operatorname{dim} W_{D}^{d+g+1} \geq 2$. However, the inclusions $0=W_{D}^{d+g-1} \subset W_{D}^{d+g} \subset W_{D}^{d+g+1}$ are all of codimension $\leq 1$ (they differ by the vanishing of one coefficient in the Laurent expansion at $P_{\infty}$ ); hence $s$ and $t$ are indeed a basis of $W_{D}^{d+g+1}$, and their degrees must be as claimed. Any other choice of $s^{\prime}, t^{\prime}$ is of the form $s^{\prime}=a s, t^{\prime}=a^{\prime} t+b s$, with $a, a^{\prime}, b \in \mathbb{K}$ and $a, a^{\prime} \neq 0$; the last assertion follows easily.

The next two propositions describe direct sum decompositions that occur in higher degrees, akin to the decompositions defining semi-typical and typical divisors. These propositions essentially mean that semi-typicality describes the initial ideal of $I_{D}$ (in the sense of Gröbner bases) with respect to the term order induced from the degree (equivalently, $v_{P_{\infty}}$ ), while typicality gives more precise information about the generators $\{s, t\}$ of $I_{D}$ and the relations between them. This information is related to the initial part of the module of first syzygies, since it describes how both generators and relations of $I_{D}$ interact with the valuation at $P_{\infty}$.

Proposition 2.4. Let $D$ be a semi-typical divisor. Then:

(1) For all $j \geq-1$, the divisor $(d+g+j) P_{\infty}-D$ is nonspecial (i.e., has trivial $\left.H^{1}\right)$. Equivalently, by Riemann-Roch, we have $\operatorname{dim} W_{D}^{d+g+j}=j+1$.

(2) For all $j \geq-1$, we have a direct sum $W_{D}^{d+g+j} \oplus W^{d+g-1}=W^{d+g+j}$.

Conversely, let $D$ be a good divisor with $\operatorname{deg} D=d \geq g$, and suppose we know for a single value of $j \geq g-1$ that $W_{D}^{d+g+j}+W^{d+g-1}=W^{d+g+j}$ (without necessarily knowing that the sum is direct). Then $D$ is in fact semi-typical, and statement (2) holds for all $j \geq-1$.

Proof. The statement on dimensions in part (1) is true for $j=-1$ by assumption. Thus $D^{\prime}=(d+g-1) P_{\infty}-D$ is nonspecial. Adding a positive multiple of $P_{\infty}$ preserves the property of being nonspecial, so we obtain part (1) for all $j$. Part (2) follows by counting dimensions as usual, since $W_{D}^{d+g+j} \cap W^{d+g-1}=W_{D}^{d+g-1}=0$.

As for the converse, the inequality $j \geq g-1$ ensures that $\operatorname{dim} W_{D}^{d+g+j}=j+1$, while $\operatorname{dim} W^{d+g-1}=d$ and $\operatorname{dim} W^{d+g+j}=d+j+1$. Thus the only way the inclusion $W_{D}^{d+g+j}+W^{d+g-1} \subset W^{d+g+j}$ can be an equality is if $W_{D}^{d+g+j} \cap W^{d+g-1}=0$, which implies that $D$ is semi-typical. 
Proposition 2.5. Let $D$ be a typical divisor, with $s, t$ as in the definition. Then for all $i \geq 0$, we have the direct sums

$$
\begin{gathered}
s W^{2 g} \oplus t W^{2 g-1+i} \oplus W^{d+g-1}=W^{d+3 g+i}, \\
s W^{2 g+i} \oplus t W^{2 g-1} \oplus W^{d+g-1}=W^{d+3 g+i}, \\
s W^{2 g} \oplus t W^{2 g-1+i}=s W^{2 g+i} \oplus t W^{2 g-1}=W_{D}^{d+3 g+i} .
\end{gathered}
$$

Conversely, given a good divisor $D$ with $\operatorname{deg} D=d \geq g$, and elements $s \in W_{D}^{d+g}, t \in$ $W_{D}^{d+g+1}$ such that one of (2.7) or (2.8) is satisfied (just with a sum - not necessarily with a direct sum) for a single value of $i \geq 0$, then $D$ is typical; hence $\{s, t\}$ is an IGS for D, and equations (2.7)-(2.9) hold for all $i$.

Proof. We know that $D$ is also semi-typical, so from part (2) of Proposition 2.4 (with $j=2 g+i)$ it is sufficient to show (2.9). We have inclusions $s W^{2 g}+t W^{2 g-1+i} \subset$ $W_{D}^{d+3 g+i}$ and $s W^{2 g+i}+t W^{2 g-1} \subset W_{D}^{d+3 g+i}$. The dimensions match up correctly as though we had equality with direct sums. To prove equality, we need to show that $s W^{2 g} \cap t W^{2 g-1+i}=s W^{2 g+i} \cap t W^{2 g-1}=0$. The proofs are similar, so we will only show that the second intersection is zero. If $u \in W^{2 g+i}$ satisfies $s u \in t W^{2 g-1} \subset$ $W^{d+3 g}$, then $\operatorname{deg} s+\operatorname{deg} u \leq d+3 g$. Since $\operatorname{deg} s=d+g$, we deduce that $\operatorname{deg} u \leq 2 g$, so in fact $s u \in s W^{2 g} \cap t W^{2 g-1}$, which we know to be zero from Proposition 2.3

Let us now prove the converse statement. Suppose for one $i$ that (2.7), say, holds (the proof in the case of (2.8) is similar). Then, by counting dimensions as in part (1) of the proof of Proposition 2.3, we obtain that the sum is direct, and that any sum made from subspaces of $s W^{2 g}, t W^{2 g-1+i}$, and $W^{d+g-1}$ will remain direct. Thus $s W^{2 g}+t W^{2 g-1}+W^{d+g-1}=s W^{2 g} \oplus t W^{2 g-1} \oplus W^{d+g-1} \subset W^{d+3 g}$, and we have equality by comparing dimensions. Thus $D$ is typical.

Corollary 2.6. Whether a good divisor $D$ with $\operatorname{deg} D=d \geq g$ is typical or semitypical depends only on the divisor class $\left[D-d P_{\infty}\right] \in \operatorname{Pic}^{0}(C)$; in particular, it depends only on the class $[D] \in \operatorname{Pic}^{d} C$.

Proof. Suppose $D^{\prime}$ is another good divisor of degree $d^{\prime} \geq g$ that maps to the same element of $\operatorname{Pic}^{0}(C)$. This means that there exists a nonzero element $u \in \mathbb{K}(C)$ of the function field whose full divisor (including $\left.P_{\infty}\right)$ is $\operatorname{div} u=D^{\prime}-D+\left(d-d^{\prime}\right) P_{\infty}$. It then follows that $W_{D^{\prime}}^{d^{\prime}+j}=u W_{D}^{d+j}$ for all $j$, so (taking $j=g-1$ ) we see that $D^{\prime}$ is semi-typical if and only if $D$ is.

Now suppose that $D$ is typical, and let $s, t$ be as in the definition. Define $s^{\prime}=$ $u s \in W_{D^{\prime}}^{d^{\prime}+g}$ and $t^{\prime}=u t \in W_{D^{\prime}}^{d^{\prime}+g+1}$. We have $s^{\prime} W^{2 g}+t^{\prime} W^{2 g-1}=u W_{D}^{d+3 g}$ by Proposition 2.3, and this last space is equal to $W_{D^{\prime}}^{d^{\prime}+3 g}$. We also know that $D^{\prime}$ is semi-typical, by the preceding paragraph, so $W_{D^{\prime}}^{d^{\prime}+3 g}+W^{d^{\prime}+g-1}=W^{d^{\prime}+3 g}$ by Proposition 2.4. Hence $s^{\prime} W^{2 g}+t^{\prime} W^{2 g-1}+W^{d^{\prime}+g-1}=W^{d^{\prime}+3 g}$, so $D^{\prime}$ is also typical, as desired.

We mention for completeness a characterization of typical divisors, phrased in terms of the degree zero line bundle $\mathcal{L}=\mathcal{O}_{C}\left(d P_{\infty}-D\right)$. The following is Proposition 3.2 of [KM16], and is proved by techniques similar to those of Proposition 2.9 in this article. Note that the first two conditions below essentially say that $\mathcal{L}$ and $\mathcal{L}^{-1}$ are semi-typical. 
Proposition 2.7. With the above notation, $D$ is typical if and only if the following three conditions hold: (i) $H^{0}\left(C, \mathcal{L}\left((g-1) P_{\infty}\right)\right)=0$, (ii) $H^{0}\left(C, \mathcal{L}^{-1}\left((g-1) P_{\infty}\right)\right)=0$, and (iii) $\mathcal{L}\left((g+1) P_{\infty}\right)$ is base point free.

We now turn to the sense in which "most" divisors are typical (hence also semitypical). More precisely, we consider divisor classes (equivalently, isomorphism classes of line bundles) in $\operatorname{Pic}^{d} C$; due to the presence of the rational point $P_{\infty}$, we can identify $\operatorname{Pic}^{d} C$ with $\operatorname{Pic}^{0} C$, and hence with the $g$-dimensional Jacobian variety of $C$. In this setting, a statement about "most" divisor classes or line bundles will mean a statement that holds for all elements of $\operatorname{Pic}^{d} C$ outside a finite union of "bad" subvarieties of dimension $\leq g-1$. This can all be studied over $\overline{\mathbb{K}}$, without changing the dimension of the appropriate bad subvarieties.

Moreover, at the expense of eliminating a few more subvarieties of dimension $g-1$, we can restrict, in the case $d \geq g$, to classes of good divisors. The way to see this is to fix a good divisor $E$, with $\operatorname{deg} E=d-g$, and then to represent any divisor class, identified with a line bundle $\mathcal{L}$ of degree $d$, by an effective divisor $D^{\prime}+E$, with $\operatorname{deg} D^{\prime}=g$; this is possible because $H^{0}(C, \mathcal{L}(-E)) \neq 0$. Then we eliminate from consideration the divisor classes represented by $E+D^{\prime}$ as $D^{\prime}$ varies over divisors of the form $P_{\infty}+Q_{1}+\cdots+Q_{g-1}$, which describe a $(g-1)$-dimensional subset of $\operatorname{Pic}^{d} C$ as the $Q_{i}$ vary. Extensions and variations of this argument give rise to the following results, which we have referred to earlier, and which we will use to control the size of the locus of nontypical divisors.

Lemma 2.8. In the following statements, "most elements $\mathcal{L} \in \mathrm{Pic}^{d} C$ " refers to all but a finite union of at most $(g-1)$-dimensional subvarieties, as discussed above.

(1) If $d \leq g-1$, then most elements $\mathcal{L} \in \operatorname{Pic}^{d} C$ satisfy $H^{0}(C, \mathcal{L})=0$.

(2) Most elements $\mathcal{L} \in \mathrm{Pic}^{g+1} C$ are base point free, with $\operatorname{dim} H^{0}(C, \mathcal{L})=2$.

Proof. We include the proof of this standard result for completeness. As noted above, we can work over $\overline{\mathbb{K}}$. If $d \leq g-1$, then an $\mathcal{L}$ with nonzero $H^{0}$ must contain an effective divisor in its class, i.e., $\mathcal{L} \cong \mathcal{O}_{C}\left(Q_{1}+\cdots+Q_{d}\right)$, which varies in a $d$-dimensional subvariety as the $Q_{i}$ vary. This proves statement (1) above.

As for statement (2), it follows from Riemann-Roch that every $\mathcal{L} \in \operatorname{Pic}^{g+1} C$ has $\operatorname{dim} H^{0}(C, \mathcal{L}) \geq 2$. If such an $\mathcal{L}$ is not base point free, there exists $P \in C(\overline{\mathbb{K}})$ such that $\operatorname{dim} H^{0}(C, \mathcal{L}(-P))=\operatorname{dim} H^{0}(C, \mathcal{L}) \geq 2$; hence $\operatorname{dim} H^{1}(C, \mathcal{L}(-P)) \geq 1$. Writing $\omega$ for the canonical bundle, we conclude that the degree $g-2$ line bundle $\omega \otimes \mathcal{L}^{-1}(P)$ has nontrivial $H^{0}$, hence that $\omega \otimes \mathcal{L}^{-1}(P) \cong \mathcal{O}_{C}\left(Q_{1}+\cdots+Q_{g-2}\right)$. Hence $\mathcal{L} \cong \omega\left(P-Q_{1}-\cdots-Q_{g-2}\right)$, and the family of such $\mathcal{L}$ has dimension $g-1$ as $P$ and the $Q_{i}$ vary. Moreover, if $\operatorname{dim} H^{0}(C, \mathcal{L}) \geq 3$, then for every $P \in C(\overline{\mathbb{K}})$, we again have $\operatorname{dim} H^{0}(C, \mathcal{L}(-P)) \geq 2$, which cannot happen for most $\mathcal{L}$ (even for one $P$ depending on $\mathcal{L}$, as we have just seen).

We note that the above reasoning generalizes to show that if $d \geq g+1$, then most choices of $\mathcal{L} \in \operatorname{Pic}^{d} C$ are base point free, with $\operatorname{dim} H^{0}(C, \mathcal{L})=d+1-g$.

Proposition 2.9. Let $d \geq g \geq 1$. Then most elements $\mathcal{L} \in \operatorname{Pic}^{d} C$ are typical and hence also semi-typical. The word "most" here has the same meaning as in Lemma 2.8.

Proof. As remarked earlier, we can restrict to the situation when $\mathcal{L}=\mathcal{O}_{C}(D)$, with $D$ a good divisor. It is convenient to show first that $\mathcal{L}$ is semi-typical, even though 
this is implied by the full result. Indeed, as $\mathcal{L}$ varies in $\operatorname{Pic}^{d} C$, the line bundle $\mathcal{O}_{C}\left((d+g-1) P_{\infty}-D\right)=\mathcal{L}^{-1}\left((d+g-1) P_{\infty}\right)$ varies in $\mathrm{Pic}^{g-1} C$. Thus (for most $\mathcal{L})$ it has no global sections, by Part (1) of Lemma 2.8, and hence $D$ is semi-typical.

We now use Part (2) of the above lemma to conclude that, for most $D$, the space $W_{D}^{d+g+1}$ is two-dimensional and base point free. Thus any basis $\{s, t\}$ of $W_{D}^{d+g+1}$ is an IGS. We can control the degrees of $s$ and $t$ so as to obtain $s, t$ as in Definition 2.2. Indeed, we already have $W_{D}^{d+g-1}=0$, so necessarily $\operatorname{dim} W_{D}^{d+g}=1$, and we can choose (any nonzero) $s \in W_{D}^{d+g} \subsetneq W_{D}^{d+g+1}$, and $t \in W_{D}^{d+g+1}$, with $t \notin W_{D}^{d+g}$. It then follows that $\operatorname{deg} s=d+g$ and $\operatorname{deg} t=d+g+1$, and we have obtained our desired $\{s, t\}$ which is an IGS for $W_{D}^{d+g+1}$.

It is immediate that $s W^{2 g}+t W^{2 g-1} \subset W_{D}^{d+3 g}$, and our next goal is to show that the above inclusion is an equality for most $D$. This is similar to the proof of Lemma 4.10 in [KM07, and is in essence the base point free pencil trick. We have as usual $\operatorname{dim} s W^{2 g}=\operatorname{dim} W^{2 g}=g+1$, and similarly $\operatorname{dim} t W^{2 g-1}=g$, while $\operatorname{dim} W_{D}^{d+3 g}=2 g+1$; it thus suffices to show that $s W^{2 g} \cap t W^{2 g-1}=0$. Write div $s=$ $D+A$ and $\operatorname{div} t=D+B$, where $A$ and $B$ are effective divisors of degrees $g$ and $g+1$, respectively (recall that $\operatorname{div} s$ and $\operatorname{div} t$ ignore the poles at $P_{\infty}$ ). Since $\{s, t\}$ is an IGS, $A$ and $B$ are disjoint. We thus have $s W^{2 g} \cap t W^{2 g-1}=W_{D+A}^{d+3 g} \cap W_{D+B}^{d+3 g}=$ $W_{D+A+B}^{d+3 g}$. Via division by the product $s t$, which introduces additional poles at $2 D+A+B$, we have that $W_{D+A+B}^{d+3 g}$ is isomorphic to $H^{0}\left(\mathcal{O}_{C}\left((g-d-1) P_{\infty}+D\right)\right)$. But this last space is the divisor of a line bundle of degree $g-1$, and hence is zero for most $D$. Hence $s W^{2 g}+t W^{2 g-1}=W_{D}^{d+3 g}$, as desired.

At this point, we know that $s W^{2 g}+t W^{2 g-1}+W^{d+g-1}=W_{D}^{d+3 g}+W^{d+g-1} \subset$ $W^{d+3 g}$, and we wish to show equality to conclude that $D$ is typical. As usual, we count dimensions: the space $W_{D}^{d+3 g}$ has codimension $d$ inside $W^{d+3 g}$, and $\operatorname{dim} W^{d+g-1}=d$, so it suffices to point out that $W_{D}^{d+3 g} \cap W^{d+g-1}=W_{D}^{d+g-1}$, which is zero since we already know that $D$ is semi-typical.

When $\mathbb{K}$ is a finite field with $q$ elements, one can give a precise quantitative bound of the probability that a random divisor class fails to be typical or semi-typical. Qualitatively, the expected probability is $O(1 / q)$ (for fixed $g$ ), since the nontypical divisors lie on a proper subvariety, but finding the implied constant takes some work. The following result is Theorem 3.3 of [KM16]. The proof there uses rather different techniques from the ones in this article, based on bounding the number of points on certain Brill-Noether loci via the Weil bounds for zeta and L-functions of curves. The probabilities given below are very small for the values of $g$ and $q$ one is likely to encounter in applying the results of this article; see Remark 4.3 below.

Theorem 2.10. Suppose that $g \geq 2$ and that $\mathbb{K}$ is a finite field with $q$ elements, with $q \geq 16^{g}$. Let $\mathcal{L}$ be a uniformly randomly chosen element of $\operatorname{Pic}^{d} C$ (the precise value of $d$ does not matter, since we can always shift by a multiple of $P_{\infty}$, as in Corollary 2.6).

(1) The probability that $\mathcal{L}$ is not semi-typical is at most $1.7 / q$.

(2) The probability that $\mathcal{L}$ is not typical is at most $\left(16^{g} \cdot g+3.4\right) / q$.

(3) The probability that $\mathcal{L}$ and $\mathcal{L}^{-1}$ (more accurately, replace $\mathcal{L}^{-1}$ by $\mathcal{L}^{-1}\left(N P_{\infty}\right)$ for some large $N)$ are not both typical is at most $\left(16^{g} \cdot 2 g+3.4\right) / q$.

Example 2.11. We illustrate semi-typical and typical divisors in the setting of $C_{3,4}$ curves, and relate our definitions to the constructions in ASKM07. Recall 
that a $C_{3,4}$ curve $C$ has genus $g=3$, and is given by an affine equation for the open set $C-\left\{P_{\infty}\right\}$ of the form $f(x, y)=y^{3}-x^{4}+\sum_{3 i+4 j<12} c_{i j} x^{i} y^{j}=0$; here $\mathcal{R}=\mathbb{K}[x, y] /\langle f(x, y)\rangle$, with $\operatorname{deg} x=3$ and $\operatorname{deg} y=4$. Any given $W^{N}$ is spanned by the first few monomials from the ordered list $1, x, y, x^{2}, x y, y^{2}, x^{3}, x^{2} y, x y^{2}, \ldots$ of degrees $0,3,4,6,7,8,9,10,11, \ldots$; the next monomial, of degree 12 , can be either $y^{3}$ or $x^{4}$, and for each larger $N$ we continue the list at the $N$ th step by choosing once and for all a monomial $x^{i} y^{j}$ with $3 i+4 j=N$, for example by limiting to $j \leq 2$. The resulting monomials $x^{2+i}, x^{1+i} y, x^{i} y^{2}, \ldots$ for $i \geq 0$ give elements of $\mathcal{R}$ of all degrees starting with $2 g=6$. The dimensions of $W^{N}$ for $N=0,1,2,3, \ldots$ are respectively $1,1,1,2,3,3,4,5,6,7, \ldots$; for example, $W^{4}=W^{5}=\mathbb{K} \cdot 1+\mathbb{K} \cdot x+\mathbb{K} \cdot y$, and for $N \geq 5=2 g-1, \operatorname{dim} W^{N}=N-2$, illustrating the first line of (2.5).

On such a curve, let $D$ be a good divisor of degree 3. In Proposition 2.1 and Equation (2) on page 310 of ASKM07, we asserted that the ideal $I_{D}$ was "typically" generated by two elements $F=x^{2}+a y+b x+c \in W_{D}^{6}$ and $G=x y+d y+e x+f \in W_{D}^{7}$, which play the roles of $s, t$ in Definition 2.2. We also asserted that "typically" $a \neq 0$. Proposition 2.12 below shows that the existence of $F, G$ as above, with $a \neq 0$, is precisely equivalent to having $D$ be typical, according to the definition in this article. The idea is that with $F, G$ as above, the ideal $I_{D}$ contains an element $H=a^{-1}(y F-x G)=y^{2}+\cdots \in W_{D}^{8}$. It follows that $I_{D}$ contains the list of elements $F, G, H, x F, x G, x H, x^{2} F, \ldots$, of degrees $6,7,8,9, \ldots$, where $6=d+g$; moreover, $W_{D}^{5}=0$ (accepting for the moment that such a $D$ is semi-typical), while $W_{D}^{N}$ for $N \geq 6$ has as a basis the first $N-5$ elements of $I_{D}$ from the above list. This illustrates the second line of (2.5).

Here is our characterization of typical and semi-typical divisors on $C_{3,4}$ curves.

Proposition 2.12. Let $D$ be a good divisor of degree 3 on a $C_{3,4}$ curve.

(1) $D$ is semi-typical if and only if there exist elements $F, G, H \in I_{D}$ of degrees $6,7,8$. After rescaling $F, G, H$ by nonzero elements of $\mathbb{K}$ (to make them "monic"), and possibly replacing $G$ by $G-\lambda F$ for some $\lambda \in \mathbb{K}$ (to eliminate the $x^{2}$ term), we can assume that $F=x^{2}+a y+b x+c \in W_{D}^{6}, G=$ $x y+d y+e x+f \in W_{D}^{7}$, and $H=y^{2}+\cdots \in W_{D}^{8}$.

(2) $D$ is typical if and only if there exist elements $F, G$ as above, with $a \neq 0$. In that case, we can take $H=a^{-1}(y F-x G)$.

Proof. Semi-typicality implies the existence of $F, G, H$ as above because of our control over the dimensions of $W_{D}^{N}$ in part (1) of Proposition 2.4. Conversely, the existence of $F, G, H$ ensures that $W_{D}^{8}+W^{5}$ contains elements with leading terms $y^{2}, x y, x^{2}, y, x, 1$, hence a "triangular" basis for $W^{8}$. We can hence apply the converse statement in Proposition 2.4.

As for typicality, take the following bases for $F W^{6}, G W^{5}$, and $W^{5}$, respectively: $\left\{F, x F, y F, x^{2} F\right\},\{G, x G, y G\}$, and $\{1, x, y\}$. Reordering all these elements and performing a harmless "elementary operation" in linear algebra, we see that the subspace $F W^{6}+G W^{5}+W^{5} \subset W^{12}$ is spanned by the set of elements $\left\{1, x, y, F, G,(y F-x G), x F, x G, y G, x^{2} F\right\}$. The leading terms of these elements are respectively $1, x, y, x^{2}, x y, a y^{2}, x^{3}, x^{2} y, x y^{2}, x^{4}$, and hence our set is "triangular" in terms of the obvious basis for $W^{12}$. Writing this in matrix form, we obtain a triangular matrix with almost all diagonal entries equal to 1 , except for a single diagonal entry of $a$ in the column corresponding to $y F-x G=a y^{2}+\cdots$; thus our set generates all of $W^{12}$ if and only if $a \neq 0$. 


\section{OPERATIONS ON TYPICAL AND SEMI-TYPICAL DIVISORS}

We now investigate how typicality and semi-typicality allow us to describe "generically correct" algorithms for operations on divisors, that succeed, roughly speaking, when the input and/or output is typical (or sometimes semi-typical), and certify both success and (semi-)typicality of the input and/or output. As a running example, we illustrate our general constructions in the setting of of $C_{3,4}$ curves, thereby making the connection with ASKM07.

We adopt the following conventions in this section:

(1) All letters describing divisors (such as $D, D^{\prime}, E$, etc.) refer to good divisors, unless otherwise specified;

(2) We will also use the corresponding lowercase letter to refer to the degree of the divisor, so $\operatorname{deg} D=d, \operatorname{deg} D^{\prime}=d^{\prime}, \operatorname{deg} E=e$, etc.;

(3) We will always assume that these degrees are $\geq g$, and that $g \geq 1$.

The context in which we will later use such divisors is that a $D$ of the above type represents the divisor class $\left[D-d P_{\infty}\right] \in \operatorname{Pic}^{0}(C)$. Conversely, every element of $\operatorname{Pic}^{0}(C)$ can be written as $\left[D-g P_{\infty}\right]$ for some effective $\mathbb{K}$-rational divisor $D$ of degree $g$, which a priori may have $P_{\infty}$ in its support, i.e., not be good. When $D$ is good, it is well known (and basically tautological) that the choice of such a $D$ is unique when $D$ is reduced; in Proposition 3.5. we recall the definition of a reduced divisor, and later show in Corollary 3.7 that typical divisors are always reduced. Hence typical elements of $\operatorname{Pic}^{0}(C)$ have a unique representation by a good divisor $D$ of degree $g$, and we do not need to go through the more elaborate tests for equality used in the general algorithms of [KM04, KM07]. We still need divisors of degrees $d \geq g$ to represent various intermediate results in our algorithms, so we carry out the discussion below for general $d$.

3.1. Addition of two divisors by taking an intersection. The first question we study concerns taking the intersection of two spaces $W_{D}^{N}$ and $W_{D^{\prime}}^{N}$. In most cases, one expects $D$ and $D^{\prime}$ to be disjoint, so the intersection should be the space $W_{D+D^{\prime}}^{N}$, or at least our calculation should be able to detect when this is indeed the case. The following result is straightforward.

Proposition 3.1. Suppose given spaces $W_{D}^{N}$ and $W_{D^{\prime}}^{N}$ as above, viewed as subspaces of $W^{N}$, and suppose that $N \geq d+d^{\prime}+g-1$. Compute the intersection $W_{D}^{N} \cap W_{D^{\prime}}^{N}$ in a way that simultaneously yields the subspace $\hat{W}=W_{D}^{d+d^{\prime}+g-1} \cap W_{D^{\prime}}^{d+d^{\prime}+g-1}$. If $\hat{W}=0$, then in fact $D$ and $D^{\prime}$ are disjoint, the intersection above correctly computes $W_{D+D^{\prime}}^{N}$, and $D+D^{\prime}$ is semi-typical.

Conversely, if $D$ and $D^{\prime}$ are disjoint, and $D+D^{\prime}$ is semi-typical, then the subspace $\hat{W}$ will indeed be zero, and hence $W_{D}^{N} \cap W_{D^{\prime}}^{N}=W_{D+D^{\prime}}^{N}$, as desired.

Proof. Write $E=\operatorname{lcm}\left(D, D^{\prime}\right)$; thus $W_{D}^{n} \cap W_{D^{\prime}}^{n}=W_{E}^{n}$ for all values of $n$, including both $n=N$ and $n=d+d^{\prime}+g-1$. The fact that $\hat{W}=W_{E}^{d+d^{\prime}+g-1}=0$ forces $e=\operatorname{deg} E \geq d+d^{\prime}$, from which we deduce that $E=D+D^{\prime}$ and that $D$ and $D^{\prime}$ are disjoint. The first result follows. As for the converse, disjointness of $D$ and $D^{\prime}$ means that $E=D+D^{\prime}$. This divisor is semi-typical, so $\hat{W}=0$.

We now discuss how one can effectively carry out linear algebra computations in subspaces of $W^{N}$, such as computing the intersections in the above proposition; this generalizes the presentation in ASKM07. Elements of $W^{N}$, for sufficiently 
large $N$, are represented as column vectors in $\mathbb{K}^{N+1-g}$ with respect to some basis of "monomials" in $\mathcal{R}$, ordered by degree. A subspace such as $W_{D}^{N} \subset W^{N}$ is represented as a matrix whose columns form a basis for $W_{D}^{N}$. When possible, we convert the basis to column-echelon form, so that the columns represent a basis for $W_{D}^{N}$ in order of increasing degree; this is illustrated in equation (3.2) in Example 3.2 below.

For computing the intersection, it is useful to set up a specific isomorphism between the quotient $\mathcal{R} / I_{D}$ (which was called $\mathcal{A}$ in Section 3 of [ASKM07]) and the vector space $W^{d+g-1}$. Since $D$ is semi-typical, we know that for $N \geq d+g-1$, the subspace $W^{N} \subset \mathcal{R}$ surjects onto $\mathcal{R} / I_{D}$, with kernel $W_{D}^{N}$. Moreover, $W^{N}=$ $W_{D}^{N} \oplus W^{d+g-1}$, so we can therefore identify $\mathcal{R} / I_{D}$ with $W^{d+g-1}$, and we have a compatible family of vector space isomorphisms

$$
W^{N} / W_{D}^{N} \cong \mathcal{R} / I_{D} \cong W^{d+g-1}, \quad \text { for all } N \geq d+g-1 \text {. }
$$

Concretely, the composition $W^{N} \rightarrow W^{N} / W_{D}^{N} \cong W^{d+g-1}$ amounts to taking elements of $W^{N}$, viewed as column vectors, and reducing the columns with respect to the columns of the matrix describing a basis for $W_{D}^{N}$ mentioned above. This reduces everything to an element of $W^{d+g-1}$, i.e., to a vector in $\mathbb{K}^{d}$.

Let us denote by $r: W^{N} \rightarrow W^{d+g-1}$ the resulting reduction map $\bmod D$. We can now compute the intersection $W_{D}^{N} \cap W_{D^{\prime}}^{N}$ as the kernel of the composite $\operatorname{map} W_{D^{\prime}}^{N} \hookrightarrow W^{N} \stackrel{r}{\rightarrow} W^{d+g-1}$. This composite map can be represented by a $d \times\left(N-d^{\prime}-g+1\right)$ matrix, which we shall call $M$; an equivalent matrix is called $M^{\prime}$ in Section 6 of ASKM07. One can compute $M$ as the product of the matrix for $r$ by the matrix whose columns give a basis for $W_{D^{\prime}}^{N}$; alternatively, take the matrix for $W_{D^{\prime}}^{N}$, and reduce each column (modulo $W_{D}^{N}$ ) to obtain columns describing the corresponding images (i.e., the reductions) in $W^{d+g-1}$.

Example 3.2. We illustrate the above in the $C_{3,4}$ case. The basis of "monomials" begins with $1, x, y, x^{2}, \ldots$, as we saw in Example 2.11. Let $D$ be semi-typical of degree 3, with elements $F, G, H$ as in Proposition 2.12. The columns of the matrix representing $W_{D}^{N}$ will then encode the echelon basis $F, G, H, x F, x G, x H, x^{2} F, \ldots$ For example, when $N=10$, then the basis of $W_{D}^{10}$ is $\{F, G, H, x F, x G\}$. Write $F=x^{2}+a y+b x+c, G=x y+d y+e x+f$, and $H=y^{2}+p x y+q x^{2}+r y+s x+t$ for certain $p, q, \ldots, t \in \mathbb{K}$. (In the typical case, $H=a^{-1}(y F-x G)$, so one can express $p, q, \ldots, t$ in terms of $a, b, \ldots, f$.) We thus obtain the following echelon form matrix which describes $W_{D}^{10}$ :

$$
\left(\begin{array}{lllll}
c & f & t & 0 & 0 \\
b & e & s & c & f \\
a & d & r & 0 & 0 \\
1 & 0 & q & b & e \\
0 & 1 & p & a & d \\
0 & 0 & 1 & 0 & 0 \\
0 & 0 & 0 & 1 & 0 \\
0 & 0 & 0 & 0 & 1
\end{array}\right), \quad \text { each row representing the coefficient of } \begin{gathered}
1 \\
x^{2} \\
x y \\
y^{2} \\
x^{3} \\
x^{2} y
\end{gathered} .
$$

The first three columns of the above matrix describe of course $W_{D}^{8}$.

(For general curves, with $D$ semi-typical, we would take generators for $W_{D}^{N}$ of degrees $d+g, d+g+1, \ldots, N$, provided $N \geq d+g-1$; the extreme case $N=d+g-1$ would correspond to an empty matrix. The columns of the matrix have their lowest 
nonzero entries in rows $d+1, d+2, \ldots, N+1-g$, because row $d+1$ corresponds to a degree $d+g$ element in the $(d+1)$-dimensional space $W^{d+g}$.)

We now illustrate the computation of an intersection as in Proposition 3.1. We first explain the reduction map $r: W^{10} \rightarrow W^{5}$, where $d+g-1=3+3-1=5$ in our setting, and the 3-dimensional space $W^{5}$ (which equals $W^{4}$ here) has basis $\{1, x, y\}$. Given an element of $W^{10}$, represented by a column vector $v \in \mathbb{K}^{8}$, we obtain its reduction by successively subtracting from $v$ multiples of the columns of the matrix in (3.2), from the rightmost column to the leftmost, in order to eliminate the lowest entries of $v$ from the bottom up. One is left with a reduced vector with only three possibly nonzero entries at the top. We identify the corresponding element of $W^{5}$ with the column vector in $\mathbb{K}^{3}$ consisting of these top three entries.

To compute the intersection $W_{D}^{10} \cap W_{D^{\prime}}^{10}$, suppose given the analogous matrix for $W_{D^{\prime}}^{10}$, in terms of the coefficients of $F^{\prime}=x^{2}+a^{\prime} y+b^{\prime} x+c^{\prime}, G^{\prime}=y^{2}+d^{\prime} y+\cdots$, and so forth. The matrix $M$ defined above is obtained by reducing the columns of the matrix for $W_{D^{\prime}}^{10}$. Thus the columns of $M$ give the reductions of $F^{\prime}, G^{\prime}, H^{\prime}, x F^{\prime}, x G^{\prime}$ modulo the columns of the matrix for $W_{D}^{10}$ in (3.2). This yields

$$
M=\left(\begin{array}{ccccc}
c^{\prime}-c & f^{\prime}-f & * & * & * \\
b^{\prime}-b & e^{\prime}-e & * & * & * \\
a^{\prime}-a & d^{\prime}-d & * & * & *
\end{array}\right)
$$

The first two columns describe the reductions $F^{\prime}-F, G^{\prime}-G \in W^{5}$ of $F^{\prime}$ and $G^{\prime}$ modulo $W_{D}^{10}$. (These were called $B_{F^{\prime}}, B_{G^{\prime}}$ in Section 4 of [ASKM07].) One obtains the third column, for instance, by reducing the column representing $H^{\prime}$ to the column corresponding to $H^{\prime}-H-\left(p^{\prime}-p\right) G-\left(q^{\prime}-q\right) F \in W^{5}$, which is a reduction with respect to the first three columns of the matrix in (3.2). The last two columns are similar.

Now the kernel of $M$ corresponds to linear combinations of $F^{\prime}, G^{\prime}, H^{\prime}, x F^{\prime}, x G^{\prime}$ that belong to $W_{D}^{10} \cap W_{D^{\prime}}^{10}$. As seen in Section 6 of ASKM07, the algorithms there find $\operatorname{ker} M$ by a Gaussian elimination that assumes that the leftmost $3 \times 3$ submatrix of $M$ is invertible. This amounts to invertibility of the leftmost $3 \times 3$ minor, called $U$ in equation (15) of ASKM07]. The key point to observe is that the above $3 \times 3$ leftmost submatrix of $M$, with determinant $U$, represents a matrix whose kernel computes $W_{D}^{8} \cap W_{D^{\prime}}^{8}$. Thus invertibility of this submatrix means that $W_{D}^{8} \cap W_{D^{\prime}}^{8}=0$, and that we satisfy the condition of Proposition 3.1. Hence the computation, if successful, returns the correct result for $W_{D+D^{\prime}}^{10}$ in terms of $\operatorname{ker} M$; the way in which this kernel is computed, which essentially expresses the fourth and fifth columns of $M$ as linear combinations of the first three columns, simultaneously ends up computing "monic" elements $s \in W_{D+D^{\prime}}^{9}, t \in W_{D+D^{\prime}}^{10}$.

We have just shown the following result.

\footnotetext{
${ }^{1}$ Actually, the algorithm there also assumes that the top left $1 \times 1$ and $2 \times 2$ minors, $A_{1}$ and $D=\Delta_{12}$, are also invertible, and replaces inverting all three quantities $A_{1}, D, U$ by one field inversion combined with several multiplications. To genuinely only compute $\operatorname{ker} M$ while checking that $U \neq 0$, one can exchange rows of $M$ as needed, which does not change the kernel or the fact that $U \neq 0$. Thus, possibly after a first row exchange, one can first ensure that $A_{1} \neq 0$, then one computes $\Delta_{12}$ and $\Delta_{13}$, which are both needed anyhow for the computation. One then exchanges rows 2 and 3 if needed to ensure that $\Delta_{12} \neq 0$.
} 
Proposition 3.3. In the $C_{3,4}$ case, let $D$ and $D^{\prime}$ be typical divisors of degree 3 . Compute $s \in W^{9}, t \in W^{10}$ as in Sections 3, 4, 6, and 7 of ASKM07. If the inversion in Proposition 6.1 of ASKM07 can be carried out2, then the result correctly produces $s, t \in I_{D+D^{\prime}}$, and one deduces that $D, D^{\prime}$ were disjoint to begin with and that $D+D^{\prime}$ is semi-typical. The converse also holds.

The same argument as in Proposition 3.1 and Example 3.2 generalizes to show:

Proposition 3.4. Let $C$ be arbitrary, and consider typical divisors $D, D^{\prime}$ of degrees $d, d^{\prime}$ (both $\geq g$, as usual), described by elements $F \in W_{D}^{d+g}, G \in W_{D}^{d+g+1}$ and $F^{\prime} \in W_{D^{\prime}}^{d^{\prime}+g}, G^{\prime} \in W_{D^{\prime}}^{d^{\prime}+g+1}$. For $N \geq d+d^{\prime}+g-1$, the following algorithm will either fail or succeed, and, if it succeeds, will correctly compute $W_{D+D^{\prime}}^{N}$. The algorithm succeeds if and only if (i) $D, D^{\prime}$ were disjoint to begin with, and (ii) $D+D^{\prime}$ is semi-typical.

Algorithm:

(1) Compute column-echelon matrices whose columns represent bases for $W_{D}^{N}$ and $W_{D^{\prime}}^{N}$, respectively. For example, if $N=d+3 g+i$ with $i \geq 0$, one can start with a basis for $W_{D}^{N}$ obtained from $F W^{2 g+i} \oplus G W^{2 g-1}$ as in (2.9), write the basis as columns, and then "column reduce" the resulting matrix into echelon form; if $N<d+3 g$, one can compute the column-reduced matrix for the larger space $W_{D}^{d+3 g}$, and select the first $N-d-g+1$ columns. (Note: If $D, D^{\prime}$ are merely semi-typical, but we have access nonetheless to column-echelon bases for the spaces $W_{D}^{N}$ and $W_{D^{\prime}}^{N}$, then we can still use these spaces and proceed to the next step.)

(2) Using the matrix for $W_{D}^{N}$, reduce the columns coming from the matrix for $W_{D^{\prime}}^{N}$ to representatives in $W^{d+g-1} \cong W^{N} / W_{D}^{N}$. This yields a matrix $M$ of size $d \times\left(N-d^{\prime}-g+1\right)$, whose columns represent the reduction modulo $I_{D}$ of the basis of $W_{D^{\prime}}^{N}$, ordered by increasing degree (of the original basis element, not of the reduction).

(3) If the leftmost $d \times d$ submatrix of $M$ is not invertible (easily seen during the linear algebra, e.g., by carrying out Gaussian elimination), then return "fail". This is because the leftmost $d$ columns of $M$ represent the map from $W_{D^{\prime}}^{d+d^{\prime}+g-1}$ to $\mathcal{R} / I_{D} \cong W^{d+d^{\prime}+g-1} / W_{D}^{d+d^{\prime}+g-1} \cong W^{d+g-1}$.

(4) Otherwise, compute an echelon basis for the kernel of $M$. This will consist of column vectors of the form $(*, \ldots, 1,0, \ldots)^{\mathbf{T}}$ with at least $d$ initial entries before the final nonzero entry 1 . By taking the corresponding linear combinations of the previous ordered basis for $W_{D^{\prime}}^{N}$, convert the basis for $\operatorname{ker} M$ into a basis for $W_{D+D^{\prime}}^{N}$, ordered by increasing degree. Return "succeed", along with the basis for $W_{D+D^{\prime}}^{N}$.

3.2. Flipping a divisor. We now turn to the question of finding a complementary divisor for a given semi-typical divisor $D$. Consider a nonzero element $s \in W_{D}^{d+g}$, which is unique up to a multiplicative constant. Hence the divisor of $s$ is uniquely determined, and we have (ignoring as usual poles at $P_{\infty}$ ) that $\operatorname{div} s=D+A$, with $\operatorname{deg} A=g$. The divisor $A$ is complementary to $D$, and our goal is to compute the space $W_{A}^{N}$ for suitable $N$; we shall refer to this operation as "flipping" the divisor $D$. On the level of ideals of $\mathcal{R}$, the effect of flipping is to compute the colon ideal

\footnotetext{
${ }^{2}$ Possibly allowing as before for row operations, so the only condition that really gets checked is $U \neq 0$.
} 
$I_{A}=\left(s \mathcal{R}: I_{D}\right)$, which satisfies $I_{D} \cdot I_{A}=s \mathcal{R}$. In the Jacobian, this corresponds to replacing the class $\left[D-d P_{\infty}\right] \in \operatorname{Pic}^{0}(C)$ by its negative class $\left[A-g P_{\infty}\right]$, since the "full" principal divisor of $s$ is $\operatorname{div} s=D+A-(d+g) P_{\infty}$.

The following proposition shows that the result $A$ of flipping is a reduced divisor; thus flipping combines inverting the class of $D$ (or, more precisely, of $D-d P_{\infty}$ ) in the Jacobian, and reducing the result.

Proposition 3.5. If $D$ is semi-typical, then its flip $A$ is reduced along $P_{\infty}$, meaning that $A$ is not linearly equivalent to any divisor of the form $A^{\prime}+P_{\infty}$, with $A^{\prime}$ effective.

Proof. If $A$ were equivalent to $A^{\prime}+P_{\infty}$, then $D+A^{\prime}-(d+g-1) P_{\infty}$ would be principal, so there would exist a nonzero element $s^{\prime} \in W_{D}^{d+g-1}$, contradicting the semi-typicality of $D$.

We can compute $W_{A}^{N}$ similarly to Subsection 2.2 of [ASKM07]. Take a nonzero $t \in W_{D}^{d+g+1}$, with $\operatorname{deg} t=d+g+1$. Write $\operatorname{div} t=D+B$, with $\operatorname{deg} B=g+1$. In the typical case, we know that $s \mathcal{R}+t \mathcal{R}=I_{D}$, since $\{s, t\}$ form an IGS for $D$; equivalently, $A$ and $B$ are disjoint. In that case, we can compute

$$
\begin{aligned}
W_{A}^{N} & =\left\{\ell \in W^{N} \mid \ell I_{D} \subset s \mathcal{R}\right\}=\left\{\ell \in W^{N} \mid \ell s, \ell t \in s \mathcal{R}\right\} \\
& =\left\{\ell \in W^{N} \mid \ell t \in s W^{N+1}\right\}, \quad \text { assuming }\{s, t\} \text { an IGS for } D .
\end{aligned}
$$

As in the discussion preceding Lemma 2.4 of ASKM07, setting up a system of linear equations to solve (3.4) is wasteful. Indeed, such a system essentially computes $t W^{N} \cap s W^{N+1}$ inside the overly large space $W^{N+d+g+1}$, even though both subspaces lie inside the smaller space $W_{D}^{N+d+g+1}$, which is usually of codimension $d$ inside $W^{N+d+g+1}$. To remove the excess dimensions from consideration, we proposed in ASKM07 to carry out a "truncated" intersection after projecting to the quotient $W^{N+d+g+1} / W^{d+g-1}$. This truncation amounts computationally to ignoring the top $d$ rows of the matrix whose kernel describes the intersection in (3.4), as in Section 8 of ASKM07. We can also describe this truncated intersection conceptually as

$$
W^{\prime}=\left\{\ell \in W^{N} \mid \ell t \in s W^{N+1}+W^{d+g-1}\right\} .
$$

In practice, we will have $N=2 g-1+i$ with $i \geq 0$, so the above computation measures the extent to which the sum $s W^{2 g+i}+t W^{2 g-1+i}+W^{d+g-1}$ is not direct. The reader should compare this with equations (2.7) and (2.8): there, the "excess degree" $i$ appeared in only one of the first two summands, and the sum was direct.

Analogously to Proposition 3.1, we begin our discussion with a criterion to guarantee that the space $W^{\prime}$ is really equal to $W_{A}^{N}$. This is the result that originally led us to define typical divisors and to investigate their properties.

Proposition 3.6. Let $D$ be given with $s \in W_{D}^{d+g}$ and $t \in W_{D}^{d+g+1}$. Suppose that $\operatorname{deg} s=d+g$, $\operatorname{deg} t=d+g+1$, and $N \geq 2 g-1$. Assume further that while computing the space $W^{\prime}$ of (3.5), we also compute $\hat{W}=W^{\prime} \cap W^{2 g-1}$, and determine that $\hat{W}=0$. Then $D$ was typical to begin with, and $W^{\prime}$ is indeed equal to $W_{A}^{N}$, where $A$ is the flip of $D$, and $\operatorname{div} s=D+A$ as in our discussion. Moreover, $A$ is semi-typical.

Conversely, if $D$ is typical, then $\hat{W}$ will equal zero, and the above computation of $W_{A}^{N}$ is correct. Thus the flip of a typical divisor is always semi-typical.

Proof. Note first that $s W^{N+1} \cap W^{d+g-1}=0$, since a nonzero multiple of $s$ must have degree at least $d+g$. (Since $\operatorname{deg} s=d+g$, we also see that the divisor $A$ 
has degree $g$.) Hence $\operatorname{dim}\left(s W^{N+1}+W^{d+g-1}\right)=(N+2-g)+d$. If $\hat{W}=0$, then $t W^{2 g-1} \cap\left(s W^{N+1}+W^{d+g-1}\right)=0$. Thus $\operatorname{dim}\left(t W^{2 g-1}+s W^{N+1}+W^{d+g-1}\right)=$ $N+d+2=\operatorname{dim} W^{N+d+g+1}$. Since $N \geq 2 g-1$, it follows that $t W^{2 g-1}+s W^{N+1}+$ $W^{d+g-1} \subset W^{N+d+g+1}$, so we obtain equality. By the converse condition to (2.8) in Proposition 2.5. we obtain that $D$ is typical, as desired. Hence $\{s, t\}$ is an IGS for $D$, and (3.4) holds, so $W_{A}^{N} \subset W^{\prime}$; in particular, $W_{A}^{2 g-1} \subset \hat{W}=0$, so we deduce that $A$ is semi-typical. It remains to show that $W^{\prime} \subset W_{A}^{N}$. Suppose that $\ell \in W^{\prime}$ satisfies $t \ell=s \ell^{\prime}+\ell^{\prime \prime}$, with $\ell^{\prime} \in W^{N+1}$ and $\ell^{\prime \prime} \in W^{d+g-1}$. Then $\ell^{\prime \prime} \in t W^{N}+s W^{N+1} \subset I_{D}$, so we conclude that $\ell^{\prime \prime} \in I_{D} \cap W^{d+g-1}=W_{D}^{d+g-1}=0$ by semi-typicality. Thus $t \ell=s \ell^{\prime}$, so $\ell \in W_{A}^{N}$ by (3.4). This proves the results in the first paragraph.

The converse holds because, when $D$ is typical, if any nonzero element $\ell \in$ $W^{2 g-1}$ satisfying (3.5) existed, it would give rise to a nontrivial linear dependence between the subspaces $t W^{2 g-1}, s W^{N+1}$, and $W^{d+g-1}$, contradicting the direct sum decomposition in (2.8).

Corollary 3.7. If $D$ is a typical divisor of degree $d=g$, then $D$ is reduced in the sense of Proposition 3.5 .

Proof. The converse in Proposition 3.6 tells us that the flip $A$ of $D$ is semi-typical. Since $s \in W_{D+A}^{2 g}$, we conclude that $D$ is also the flip of $A$, so by applying Proposition 3.5 to $A$, we deduce that $D$ is reduced.

The following is the algorithm that corresponds to Proposition 3.6. We state it for general $d$, but in fact will apply it mainly when $d=g$ or $d=2 g$.

Proposition 3.8. Make the same assumptions on $D, s, t$, and $N$ as in Proposition 3.6. The following algorithm succeeds if and only if $D$ is typical, and, upon success, correctly computes $W_{A}^{N}$, and certifies that the input $D$ was typical and that the output $A$ is semi-typical.

Algorithm:

(1) Compute an $(N+2) \times(N-g+2)$ matrix $M^{\prime}$ (analogous to the last six columns of the matrix $N^{\prime}$ in Section 9 of ASKM07), whose columns describe an echelon basis for the image of $s W^{N+1}$ in $W^{N+d+g+1} / W^{d+g-1}$. (This amounts to multiplying s by each "monomial" in $W^{N+1}$ in order of increasing degree, and ignoring the d terms of "lowest degree" in each result.) It follows that reducing modulo the columns of $M^{\prime}$ implements the reduction map from the $(N+2)$-dimensional space $W^{N+d+g+1} / W^{d+g-1}$ to the $g$-dimensional space $V=W^{N+d+g+1} /\left(W^{d+g-1}+s W^{N+1}\right)$.

(2) Take a similar echelon basis for the image of $t W^{N}$ in $W^{N+d+g+1} / W^{d+g-1}$, and use the matrix $M^{\prime}$ to reduce each element of this basis into $V$. Make a new $(N-g+1) \times g$ matrix $M^{\prime \prime}$ whose columns are the reductions of these basis elements. Thus the leftmost $g$ columns of $M^{\prime \prime}$ represent the reductions of $t W^{2 g-1}$ to the space $V$.

(3) Perform Gaussian elimination on $M^{\prime \prime}$ to find its kernel, which corresponds to the space $W^{\prime}$ of (3.5). Along the way, compute $\hat{W}$ as the kernel of the leftmost $g \times g$ submatrix of $M^{\prime \prime}$. If $\hat{W} \neq 0$, then return "fail".

(4) Otherwise, compute an echelon basis for the kernel of $M^{\prime \prime}$; analogously to Proposition 3.4. this produces $N-2 g+1$ elements $\ell \in W^{N}$, ordered by degree, that satisfy equation (3.4). Return "succeed", along with these elements as a basis for $W_{A}^{N}$. 
Example 3.9. We illustrate the above algorithm on the results of Section 9 of ASKM07. In that context, the divisor that we wish to flip is written $D+D^{\prime}$, of degree $d=2 g=6$, and we know that $D+D^{\prime}$ is semi-typical (this follows from Proposition 3.3 above, when $D \neq D^{\prime}$, and from Proposition 3.17 below, when $\left.D=D^{\prime}\right)$. In particular, we know elements $s \in W_{D+D^{\prime}}^{9}$ and $t \in W_{D+D^{\prime}}^{10}$. In flipping this divisor, we have $\operatorname{div} s=D+D^{\prime}+D^{\prime \prime}$ with $\operatorname{deg} D^{\prime \prime}=g=3$, and we wish to compute $W_{D^{\prime \prime}}^{N}$ for $N=7$. We calculate $W^{\prime}$ as in (3.5) by working in the quotient space $V=W^{N+d+g+1} /\left(s W^{N+1} \oplus W^{d+g-1}\right)=W^{17} /\left(s W^{8} \oplus W^{8}\right)=W^{17} /\left(s W^{8}+W^{9}\right)$, where the last equality follows from $\mathbb{K} s+W^{8}=W^{9}$. We have $\operatorname{dim} V=3$, and we construct in that article a matrix $M^{\prime \prime}$ whose columns represent the images in $V$ of the basis $\left\{t, x t, y t, x^{2} t, x y t\right\}$ for $t W^{7}$. Thus an element of ker $M^{\prime \prime}$ describes a linear combination of $c_{0} t+c_{1} x t+\ldots$, that lies in $t W^{7} \cap\left(s W^{8} \oplus W^{8}\right)$, hence simultaneously describes a combination $\ell=c_{0}+c_{1} x+\cdots \in W^{\prime}$. Now in Proposition 9.3 of [ASKM07, we simultaneously invert two field elements $\beta_{2}$ and $\gamma_{4}$; in that context, invertibility of $\beta_{2}$ means that the first three columns of $M^{\prime \prime}$ are linearly independent. These columns represent the images of $t, t x, t y$ in $V$, where we recall that $\operatorname{span}\{1, x, y\}=W^{4}=W^{5}$, so independence of these three columns means that $W^{\prime} \cap W^{5}=0$. Hence by Proposition 3.6. we deduce (provided we are able to invert $\beta_{2}$ ) that $D+D^{\prime}$ is indeed typical, and that $W^{\prime}=W_{D^{\prime \prime}}^{7}$, as desired. Our results show that $D^{\prime \prime}$ is semi-typical, but in this case we can prove the stronger result that $D^{\prime \prime}$ is typical, provided $\gamma_{4}$ is also invertible. To see this, refer to the last paragraph of Section 9 of ASKM07, where one sees that calculating ker $M^{\prime \prime}$ produces elements of the form $F^{\prime \prime}=x^{2}+a^{\prime \prime} y+b^{\prime \prime} x+c^{\prime \prime}, G^{\prime \prime}=x y+d^{\prime \prime} y+e^{\prime \prime} x+f^{\prime \prime} \in W_{D^{\prime \prime}}^{7}$. Here, somewhat miraculously, $a^{\prime \prime}=-\gamma_{4}$, so it is invertible, and hence Proposition 2.12 tells us that $D^{\prime \prime}$ is typical, which goes beyond our result for arbitrary $C$.

We have thus shown:

Proposition 3.10. In the $C_{3,4}$ case, suppose that $s \in W_{D+D^{\prime}}^{9}$ and $t \in W_{D+D^{\prime}}^{10}$ are as given in the input of Sections 8 and 9 of ASKM07. If calculations of those sections can be carried out, including the inversion of the product $\beta_{2} \gamma_{4}$, then the final result of that calculation correctly computes the "flip" $D^{\prime \prime}$ of $D+D^{\prime}$, and it also certifies that $D^{\prime \prime}$ is typical. Conversely, if $D+D^{\prime}$ and $D^{\prime \prime}$ are both typical, then the product $\beta_{2} \gamma_{4}$ can be successfully inverted, and the calculation succeeds.

Example 3.11. We now apply Proposition 3.6 to the case of flipping a divisor of degree $d=3$ on a $C_{3,4}$ curve. This is needed in Subsection 2.3 and Section 10 of ASKM07. Consider a typical divisor $D$, described as usual in terms of $\{F, G\}$ instead of $\{s, t\}$, where $F=x^{2}+a y+b x+c \in W_{D}^{6}$ (with $\left.a \neq 0\right)$ and $G=x y+d y+$ $e x+f \in W_{D}^{7}$. We can write $\operatorname{div} F=D+A, \operatorname{div} G=D+B$ where this time we know that $A$ and $B$ are disjoint, and (3.4) holds. In our computation of the "flip" of $D$, we obviously know that $F \in W_{A}^{6}$, so our goal is to find an element $G_{1} \in W_{A}^{7}$, where $A$ is described by $\left\{F, G_{1}\right\}$; hence we wish to apply Proposition 3.6 with $N=7$. The desired element $G_{1}$ must satisfy $G_{1} G \in F W^{8}$, and as in the proof of our proposition, it is enough to know that $G_{1} G \in F W^{8}+W^{5}$, since any possible difference between $G_{1} G$ and an element of $F W^{8}$ must belong to $(F \mathcal{R}+G \mathcal{R}) \cap W^{5}=W_{D}^{5}=0$. Now in Equation (19) of ASKM07, we exhibit specific elements $G_{1} \in W^{7}, H_{1} \in W^{8}$ that satisfy $G G_{1}+F H_{1} \in W^{4}=W^{5}$, so this $G_{1}$ is our desired element. (In that equation,

\footnotetext{
${ }^{3}$ In fact, $D+D^{\prime}$ is typical if and only if $D^{\prime \prime}$ is, due to Corollary 3.13 Indeed, $D+D^{\prime}$ and $D^{\prime \prime}$ are flips of each other, up to the equivalence of Corollary 2.6
} 
we wrote $G^{\prime \prime \prime}, H$ instead of $G_{1}, H_{1}$, but we do not want to cause confusion with our notation $H$ from this article; besides, the notation $G_{1}, H_{1}$ appears in Section 5 of ASKM07 with the same meaning that we wish to use now.)

We deduce the following result.

Proposition 3.12. In the $C_{3,4}$ case, let $D$ be a typical divisor of degree 3 , described by $F, G$ as above. Compute $G_{1} \in W^{7}, H_{1} \in W^{8}$ such that $G G_{1}+F H_{1} \in W^{5}$, as described in the above paragraph. Then (i) $G G_{1}+F H_{1}=0$; (ii) $\left\{F, G_{1}\right\}$ are an IGS for the complementary divisor $A$ of $D$; (iii) $A$ is typical; (iv) the divisors of $G_{1}$ and $H_{1}$ have the form $\operatorname{div} G_{1}=A+E$ and div $H_{1}=B+E$; and (v) the divisors $A$ and $B$ are disjoint, the divisors $D$ and $E$ are disjoint, $\operatorname{deg} A=\operatorname{deg} D=3$, and $\operatorname{deg} B=\operatorname{deg} E=4$.

Proof. By the discussion preceding the proposition, we have $F \in W_{A}^{6}$ and $G_{1} \in W_{A}^{7}$, and (i) holds. Moreover, $F=x^{2}+a y+b x+c$ with $a \neq 0$, so we deduce from Proposition 2.12 that $A$ is typical. This yields (ii) and (iii). The divisor of $G_{1} \in W_{A}^{7}$ must have the form $A+E$, and $\left\{F, G_{1}\right\}$ are an IGS for $A$, so $E$ is disjoint from $D$. Finally, the divisor of $H_{1}$ follows from the fact that $F H_{1}=-G G_{1}$ has divisor $\operatorname{div} G+\operatorname{div} G_{1}=D+B+A+E$. This shows (iv) and (v), and completes the proof.

Corollary 3.13. In the $C_{3,4}$ case, a divisor $D$ is typical if and only if its "flip" $A$ is typical.

Proof. We have just seen this in case $d=3$, since both $D$ and $A$ share the same $F \in W^{6}$ with $a \neq 0$; this uses Part (2) of Proposition 2.12, For a higher degree divisor, the result follows from Corollary [2.6, since $D-d P_{\infty}$ is equivalent to a divisor of the form $D^{\prime}-3 P_{\infty}$, and $D$ and $D^{\prime}$ will have the same "flip".

For arbitrary $C$, we suspect that typicality is not preserved by flipping. However, in the situation generalizing Proposition 3.12, we are likely in practice to encounter a divisor $D$ with $\operatorname{deg} D=g$ as the result of flipping a previous divisor $\tilde{D}$; see for example Sections 10 and 11 of ASKM07, where our current triple $(\tilde{D}, D, A)$ corresponds to $\left(D+D^{\prime}, D^{\prime \prime}, D^{\prime \prime \prime}\right)$ in that article.

Proposition 3.14. Let $C$ be arbitrary. Suppose that $D$ is a semi-typical divisor with $d=g$, and take as usual $F \in W_{D}^{2 g}, G \in W_{D}^{2 g+1}$ with $\operatorname{div} F=D+A, \operatorname{div} G=$ $D+B$. Assume that $D$ was originally obtained as a successful flip of a divisor $\tilde{D}$, using the algorithm in Proposition [3.8. Suppose we now use the algorithm a second time, and it successfully computes $W_{A}^{N}$ for some $N \geq 2 g+1$. Then $A$ and $D$ are both typical, and the echelon basis for $W_{A}^{N}$ computed by our second application of the algorithm begins with the same element $F \in W_{A}^{2 g}$, and a new element $G_{1} \in W_{A}^{2 g+1}$. As a byproduct of this second application, based on (3.5) (and using $(F, G)$ for $(s, t)$ ), we also obtain an element $H_{1} \in W^{2 g+2}$ for which $G G_{1}+F H_{1} \in W^{2 g-1}$. Then the conclusions of Proposition 3.12 hold, with the slight modification that $\operatorname{deg} A=\operatorname{deg} D=g$ and $\operatorname{deg} B=\operatorname{deg} E=g+1$.

Conversely, if $D$ is obtained as the flip of $\tilde{D}$ as above, and $D$ is typical, then the second flip that computes A will succeed using the algorithm in Proposition 3.8 .

Proof. Only the first collection of statements needs proof; the converse is included in Proposition 3.8 
Upon successful completion of the computation, the divisors $\tilde{D}$ and $D$ are certified to be typical, because both the first and second uses of the algorithm are certified by Proposition 3.8. Thus $A$ is also typical by Corollary 2.6. because $A-g P_{\infty}$ is linearly equivalent to $\tilde{D}-\tilde{d} P_{\infty}$, both being linearly equivalent to $g P_{\infty}-D$ (the "negation" of the class $\left.\left[D-g P_{\infty}\right]\right)$. We have $G G_{1}+F H_{1} \in W^{2 g-1}$ from (3.5), and as usual $G G_{1}+F H_{1} \in G \mathcal{R}+F \mathcal{R}=I_{D}$, so in fact $G G_{1}+F H_{1} \in W_{D}^{2 g-1}=0$. The rest of the proof is a similarly direct adaptation of the proof of Proposition 3.12 .

3.3. Doubling a divisor. Our goal in this subsection is to compute the space $W_{2 D}^{N}$, for suitable $N$, when given a divisor $D$. For convenience, we will restrict to semi-typical $D$ of degree $d=g$. Our computation of $W_{D+D^{\prime}}^{N}$ via an intersection in Subsection 3.1 cannot be used directly with $D^{\prime}=D$. Instead of looking at elements of $W_{D^{\prime}}^{N}$ which vanish at $D$, we can proceed as in Section 5 of [ASKM07, where we set up a system of equations for $W_{2 D}^{N}$ to compute sections $\ell \in I_{D}$ whose differential $d \ell$ also vanishes at $D$. In this article, we set up exactly the same system of equations, but justify correctness of the equations from two new perspectives. We believe that both the old and the two new points of view have value, and we encourage the reader to compare the treatment here with the one in ASKM07. The following is the system of equations and the analogous algorithm to our previous article.

Proposition 3.15. Suppose that $D$ is semi-typical of degree $g$. Let $D$ be described as usual by $F \in W_{D}^{2 g}, G \in W_{D}^{2 g+1}$ (with $\operatorname{deg} F=2 g, \operatorname{deg} G=2 g+1$ ), and write $\operatorname{div} F=D+A, \operatorname{div} G=D+B$. Now suppose, similarly to Proposition 3.14, that we successfully use the flipping algorithm of Proposition 3.8 to compute the basis $\left\{F, G_{1}\right\}$ of $W_{A}^{2 g+1}$, alongside $H_{1} \in W^{2 g+2}$ for which $G G_{1}+F H_{1}=0$. As before, write $\operatorname{div} G_{1}=A+E$, $\operatorname{div} H_{1}=B+E$. At this point, the success of the flipping algorithm guarantees that $A$ is semi-typical and $D$ is typical, so in particular $A$ and $B$ are disjoint; however, unlike Proposition 3.14. we do not assume that we obtained $D$ as the successful flip of some $\tilde{D}$, so possibly A might not be typical, and we cannot assert (yet) that $D$ and $E$ are disjoint.

Let $N \geq 3 g-1$, and compute the space $W^{\prime \prime}$ defined by

$$
W^{\prime \prime}=\left\{\ell=a F+b G \in W_{D}^{N} \mid a, b \in \mathcal{R} \text { with } \ell^{\prime}:=a G_{1}-b H_{1} \in W_{D}^{N+1}\right\} .
$$

Moreover, suppose that our computation also yields $\hat{W}:=W^{\prime \prime} \cap W^{3 g-1}$. If $\hat{W}=0$, then $A$ is also typical, $W^{\prime \prime}$ correctly computes $W_{2 D}^{N}$, and $2 D$ is semi-typical.

Conversely, if $A$ is typical and $2 D$ is semi-typical, then $\hat{W}=0$, and the above algorithm succeeds and correctly computes $W_{2 D}^{N}$.

Proof. Before we begin, observe that $\ell^{\prime}=\left(G_{1} / F\right) \ell$, because $G G_{1}=-F H_{1}$; also observe that the full divisor (i.e., including $\left.P_{\infty}\right)$ of $\ell^{\prime} / \ell=G_{1} / F$ is $\operatorname{div} G_{1}-\operatorname{div} F=$ $\left(A+E-(2 g+1) P_{\infty}\right)-\left(A+D-2 g P_{\infty}\right)=E-D-P_{\infty}$. In particular, $\operatorname{deg} \ell^{\prime}=\operatorname{deg} \ell+1$ with $\operatorname{deg} \ell \leq N$, so $\ell^{\prime} \in W^{N+1}$ automatically; the significant condition on $\ell^{\prime}$ is that it should belong to $I_{D}$.

We give two proofs of our result. For the first proof, we know that $F \mathcal{R}=I_{D+A}$ and $F \mathcal{R}+G \mathcal{R}=I_{D}$. As $D$ and $E$ are not necessarily disjoint, let $A^{\prime}=\operatorname{gcd}(D, E)$, and write $D=A^{\prime}+D^{\prime}, E=A^{\prime}+E^{\prime}$, with $D^{\prime}, E^{\prime}$ disjoint. Then $F \mathcal{R}+G_{1} \mathcal{R}=$ $I_{A+A^{\prime}}$. Now an element $\ell$ belongs to $W^{\prime \prime}$ if and only if it satisfies the following conditions: (i) $\ell \in W^{N}$, or equivalently $\ell^{\prime} \in W^{N+1}$; (ii) $\ell \in I_{D}$; and (iii) $\ell G_{1}=F \ell^{\prime}$ belongs to $F I_{D}=I_{2 D+A}$. Note however that condition (ii) is equivalent to having $\ell F \in F I_{D}=I_{2 D+A}$. Thus we see that conditions (ii) and (iii) mean that $\ell$ belongs 
to a certain colon ideal, namely $\ell \in\left(I_{2 D+A}: F \mathcal{R}+G_{1} \mathcal{R}\right)=\left(I_{2 D+A}: I_{A+A^{\prime}}\right)=$ $I_{D+D^{\prime}}$. Thus $W^{\prime \prime}=W_{D+D^{\prime}}^{N}$, and $\hat{W}=W_{D+D^{\prime}}^{3 g-1}$. Now the fact that $\hat{W}=0$ forces $\operatorname{deg}\left(D+D^{\prime}\right) \geq 2 g$, which means that necessarily $D=D^{\prime}, A^{\prime}=0$, and $D, E$ are disjoint. Thus we have successfully computed $W_{2 D}^{N}$, with $W_{2 D}^{3 g-1}=0$, i.e., $2 D$ is semi-typical. Finally, to see that $A$ is typical, we argue as in Proposition 2.9 that the inclusion $F W^{2 g}+G_{1} W^{2 g-1} \subset W_{A}^{4 g}$ must be an equality. As usual, it is enough by dimension-counting to check that $F W^{2 g} \cap G_{1} W^{2 g-1}=0$. But this intersection is precisely $W_{A+D}^{4 g} \cap W_{A+E}^{4 g}=W_{A+D+E}^{4 g}$, because we know that $D$ and $E$ are disjoint. Moreover, this last space is isomorphic (via division by $G_{1}$ ) to $W_{D}^{2 g-1}=0$. After this, we again argue that $W_{A}^{4 g}+W^{2 g-1}=W^{4 g}$ since $A$ is semi-typical.

Conversely, suppose we know from the start that $A$ is typical. Then $D, E$ are necessarily disjoint, and $F \mathcal{R}+G_{1} \mathcal{R}=I_{A}$, so we immediately obtain $W^{\prime \prime}=W_{2 D}^{N}$ and $\hat{W}=W_{2 D}^{3 g-1}=0$ by semi-typicality of $2 D$. Our first proof is now complete.

Our second proof, which we generalize below, is to consider the definition of $W^{\prime \prime}$ as a system of equations for $\ell^{\prime}$ instead of for $\ell$. From our knowledge of the divisor of $\ell^{\prime} / \ell=G_{1} / F$, we see that the condition $\ell \in W_{D}^{N}$ corresponds to $\ell^{\prime} \in W_{E}^{N+1}$; hence our calculation is equivalent to computing $W^{\prime \prime \prime}=\left\{\ell^{\prime} \in W_{E}^{N+1} \mid \ell^{\prime} \in W_{D}^{N+1}\right\}=$ $W_{E}^{N+1} \cap W_{D}^{N+1}$. This intersection is $W_{D+E}^{N+1}$ precisely when $D$ and $E$ are disjoint, which can be certified by the condition $\hat{W}=0$, since this condition is equivalent to $W^{\prime \prime \prime} \cap W^{3 g}=0$, which by Proposition 3.1 allows us to conclude that $D, E$ are disjoint and that $D+E$ is semi-typical. Now $[E]=\left[D+P_{\infty}\right]$, so $[D+E]=\left[2 D+P_{\infty}\right]$, hence Corollary 2.6 implies that $2 D$ is semi-typical if and only if $D+E$ is. Finally, $W^{\prime \prime}=\left(F / G_{1}\right) W^{\prime \prime \prime}=\left(F / G_{1}\right) W_{D+E}^{N+1}=W_{2 D}^{N}$, as desired. The remaining assertions follow similarly to the first proof above. So, in essence, we use $G_{1} / F$ to move between the class of $D-g P_{\infty}$ and the equivalent class of $E-(g+1) P_{\infty}$, and we replace doubling $D$ with adding $D+E$, which can be carried out using our earlier methods. We then move back within the equivalence class to $2 D$.

We make some remarks on how one computes the space $W^{\prime \prime}$ in practice. Since $D$ is typical, we have a direct sum $W^{2 g+i} F+W^{2 g-1} G=W_{D}^{4 g+i}$ for $i \geq 0$. This allows us to proceed smoothly if $N=4 g+i \geq 4 g$, by taking all $a \in W^{2 g+i}$ and $b \in W^{2 g-1}$, but in practice we want $N=3 g+1$. In that case, in setting up a system of equations for $\ell=a F+b G$, we must restrict the possible values of pairs $(a, b) \in W^{2 g} \times W^{2 g-1}$ to ensure that $\operatorname{deg} \ell \leq N$.

Example 3.16. In the $C_{3,4}$ case, we saw in Proposition 2.12 that $W_{D}^{12}=F W^{6}+$ $G W^{5}$ has a basis $\left\{F, G, y F-x G, x F, x G, y G, x^{2} F\right\}$, ordered by degree, of which the first five elements are a basis for $W_{D}^{10}$. Hence the pairs $(a, b)$ to consider are $\mathbb{K}$-linear combinations of $\{(1,0),(0,1),(y,-x),(x, 0),(0, x)\}$. In Sections 5 and 6 of ASKM07, when $D=D^{\prime}$, we looked for elements $\ell \in W^{\prime \prime}$ of the form $\ell=$ $c_{1}^{\prime} F+c_{2}^{\prime} G+c_{3}^{\prime}(y F-x G)+c_{4}^{\prime} x F+c_{5}^{\prime} x G$. Now the corresponding $\ell^{\prime}$ is $\ell^{\prime}=c_{1}^{\prime} G_{1}-$ $c_{2}^{\prime} H_{1}+c_{3}^{\prime}\left(y G_{1}+x H_{1}\right)+c_{4}^{\prime} x G_{1}-c_{5}^{\prime} x H_{1}$, and we want to set up a system of equations that ensures that $\ell^{\prime}$ has zero image in the three-dimensional quotient $W^{11} / W_{D}^{11}$. This is exactly the kernel of the matrix $M^{\prime}$ in Section 6 of ASKM07. Moreover, the first three columns of $M^{\prime}$ correspond to taking $\ell$ to be a linear combination of $F, G, y F-x G$, or respectively to $\ell^{\prime}$ being a linear combination of $G_{1},-H_{1}, y G_{1}+$ $x H_{1}$, and invertibility of the leftmost $3 \times 3$ submatrix of $M^{\prime}$ is exactly the condition that $0=W^{\prime \prime} \cap \operatorname{span}(F, G, y F-x G)=W^{\prime \prime} \cap W_{D}^{8}$, or respectively that $0=W^{\prime \prime \prime} \cap W_{E}^{9}$. This is exactly what we need to apply Proposition 3.15 
Combining the above with Proposition 3.12 to compute first $\left\{G_{1}, H_{1}\right\}$, we obtain:

Proposition 3.17. In the $C_{3,4}$ case, let $D$ be a typical degree 3 divisor. If the computations in Sections 5, 6, and 7 of [ASKM07] can be carried out, including the inversion in Proposition 6.1 of that article, then the computation succeeds, and correctly returns $s \in W_{2 D}^{9}, t \in W_{2 D}^{10}$, if and only if $2 D$ is semi-typical. In all cases, the kernel of $M^{\prime}$ will compute the space $W_{2 D}^{10}$.

Generalizing this method from the $C_{3,4}$ case to an arbitrary curve $C$ is straightforward, once one takes into account some possibly more complicated conditions on the pairs $(a, b) \in W^{2 g} \times W^{2 g-1}$ that one wishes to consider in the system of equations. We will leave the details of a general algorithm in that case to the reader.

We now turn to our second method for doubling. This time, we begin with a divisor $\tilde{D}$ whose flip will be the divisor $D$ that we wish to double. This is analogous to combining Proposition 3.14 with the second proof in Proposition 3.15. The idea is to combine a slight extension of the flipping algorithm that produces $D$ with the ideas of Proposition 3.15, in a way that obtains both $W_{D}^{N+1}$ and $W_{E}^{N+1}$ for a suitable $N$; here $E$ is the same as in Proposition 3.15, using $\tilde{D}$ instead of $A$. The intersection $W_{D}^{N+1} \cap W_{E}^{N+1}=W_{D+E}^{N+1}$ can then be transferred back to give $W_{2 D}^{N}$.

Proposition 3.18. Let $\tilde{D}$ be a semi-typical divisor of degree $\tilde{d}$, and assume given $\tilde{s} \in W_{\tilde{D}}^{\tilde{d}+g}, \tilde{t} \in W_{\tilde{D}}^{\tilde{d}+g+1}$ as usual. Write $\operatorname{div} \tilde{s}=D+\tilde{D}$ and $\operatorname{div} \tilde{t}=E+\tilde{D}$, with $\operatorname{deg} D=g$ and $\operatorname{deg} E=g+1$. For $N \geq 3 g-1$, the following algorithm, if successful, simultaneously computes, for both the flipped divisor $D$ and its double $2 D$, the spaces $W_{D}^{N+1}$ and $W_{2 D}^{N}$. The algorithm succeeds in computing $W_{D}^{N+1}$ and $W_{2 D}^{N}$ if and only if $\tilde{D}$ is typical, and correctly certifies semi-typicality of $2 D$ whenever it holds.

Algorithm:

(1) Set up a system of equations that computes the space of pairs

$$
\tilde{W}=\left\{\left(\ell, \ell^{\prime}\right) \in W^{N+1} \times W^{N+2} \mid \tilde{t} \ell+\tilde{s} \ell^{\prime} \in W^{\tilde{d}+g-1}\right\} .
$$

Concretely, make an $(N+3) \times(2 N-2 g+5)$ matrix $\tilde{M}$ concatenating matrices similar to those produced by Steps 1 and 2 of the algorithm in Proposition 3.8. More precisely, the columns of $\tilde{M}$ consist of $N-g+3$ columns representing the images (in $W^{N+\tilde{d}+g+2} / W^{\tilde{d}+g-1}$ ) of a basis for $\tilde{s} W^{N+2}$, and of $N-g+2$ other columns representing the images of a basis for $\tilde{t} W^{N+1}$. The matrix should be computed in terms of products s̃m (respectively, $\tilde{t} m$ ) over various "monomials" $m$, indexed by degree, that give a basis for $W^{N+2}$ (respectively, $W^{N+1}$ ), and the products should themselves also be expressed in terms of a basis of monomials describing $W^{N+\tilde{d}+g+2} / W^{\tilde{d}+g-1}$. This is analogous to the entire matrix $N^{\prime}$ in Section 8 of ASKM07.

(2) Find $\operatorname{ker} \tilde{M}$ (i.e., the space $\tilde{W}$ ) in a way that simultaneously verifies that a certain $(2 g+1) \times(2 g+1)$ submatrix of $\tilde{M}$ is invertible. Specifically, detect whether the columns describing $\{\tilde{s} m \mid \operatorname{deg} m \leq 2 g\} \cup\{\tilde{t} m \mid \operatorname{deg} m \leq 2 g-1\}$ are linearly independent; note that these columns all correspond to elements of $W^{\tilde{d}+3 g} / W^{\tilde{d}+g-1} \subset W^{N+\tilde{d}+g+2} / W^{\tilde{d}+g-1}$, so only the top $2 g+1$ entries are nonzero. In practice, these columns should be placed as the leftmost columns of $\tilde{M}$, and one computes an echelon basis for $\operatorname{ker} \tilde{M}$. If these columns are not linearly independent, then return "fail". 
(3) Success at the previous step certifies that $\tilde{D}$ is typical, hence that $D$ (and also $E$ ) is semi-typical; every pair $\left(\ell, \ell^{\prime}\right) \in \tilde{W}$ actually satisfies $\tilde{t} \ell+\tilde{s} \ell^{\prime}=0$. With respect to a suitable ordering by degree, one can find an echelon basis for $\tilde{W}$ of the form $\left\{\left(\ell_{j}, \ell_{j}^{\prime}\right) \mid 1 \leq j \leq N-2 g+2\right\}$, with $\operatorname{deg} \ell_{j}=2 g-1+j$, $\operatorname{deg} \ell_{j}^{\prime}=2 g-j$. Moreover, $\left\{\ell_{1}, \ldots, \ell_{N-2 g+2}\right\}$ is a basis for $W_{D}^{N+1}$, while $\left\{\ell_{1}^{\prime}, \ldots, \ell_{N-2 g+2}^{\prime}\right\}$ is a basis for $W_{E}^{N+2}$. Discarding $\ell_{N-2 g+2}^{\prime}$, we actually have that $\left\{\ell_{1}^{\prime}, \ldots, \ell_{N-2 g+1}^{\prime}\right\}$ is a basis for $W_{E}^{N+1}$.

(4) Compute $W_{D+E}^{N+1}$ as the intersection $W_{D}^{N+1} \cap W_{E}^{N+1}$, in a way that also identifies $\hat{W}=W_{D+E}^{3 g}$ (this is easy to do with echelon bases). Then $\hat{W}=0$ if and only if $D+E$, equivalently $2 D$, is semi-typical. In practice, one should compute the intersection by computing the space of tuples $\left(c_{1}, \ldots, c_{N-2 g+1}\right)$ which satisfy $\sum_{j} c_{j} \ell_{j}^{\prime}=0 \in W^{N+1} / W_{D}^{N+1}$.

(5) For each tuple $\left(c_{j}\right)$ as above (take a basis of the space of such tuples), compute $\sum_{j} c_{j} \ell_{j} \in W_{2 D}^{N}$. The collection of such $\sum_{j} c_{j} \ell_{j}$ gives a basis for $W_{2 D}^{N}$, which can be arranged to be in echelon form due to our control of the degrees $\operatorname{deg} \ell_{j}$.

Proof. If our computation passes Step (2), we conclude that $\tilde{s} W^{2 g}+\tilde{t} W^{2 g-1}$ generates all of $W^{\tilde{d}+3 g} / W^{\tilde{d}+g-1}$, from which it follows that $\tilde{D}$ is typical and that $D, E$ are disjoint. Moreover, $D$ is semi-typical, being the flip of the typical divisor $\tilde{D}$, and $E$ is also typical by Corollary 2.6 (using the fact that the full $\operatorname{divisor} \operatorname{div}(\tilde{t} / \tilde{s})$ is $\left.E-D-P_{\infty}\right)$. Moreover, all combinations $\tilde{t} \ell+\tilde{s} \ell^{\prime}$ belong to $I_{\tilde{D}}$, so a pair $\left(\ell, \ell^{\prime}\right) \in \tilde{W}$ must satisfy $\tilde{t} \ell+\tilde{s} \ell^{\prime} \in W_{\tilde{D}}^{\tilde{d}+g-1}=0$. This proves the first assertions of (3).

The next assertions of (3) boil down to observing that projecting from $\left(\ell, \ell^{\prime}\right) \in \tilde{W}$ to the $\ell$ component is equivalent to our usual algorithm for flipping $\tilde{D}$ to find $W_{D}^{N+1}$, as in (3.5); similarly for having the $\ell^{\prime}$ compute $W_{E}^{N+2}$. Another way to see this last fact is that $\tilde{W}$ is the graph of the bijection $\ell \mapsto \ell^{\prime}=-(\tilde{t} / \tilde{s}) \ell$ between $W_{D}^{N+1}$ and $W_{E}^{N+2}$. By semi-typicality of either of these spaces, there exist corresponding "triangular" bases of elements $\ell_{j}$ or $\ell_{j}^{\prime}$ with the degrees that we claim. Hence (3) is now proved.

The intersection in (4) is $W_{D+E}^{N+1}$ as claimed, since we have already observed that $D$ and $E$ are disjoint; the comment on semi-typicality of $D+E$ is immediate, as is the fact that $2 D$ will then also be semi-typical by Corollary 2.6 . Finally, the correspondence in (5) between $\sum_{j} c_{j} \ell_{j}^{\prime} \in W_{E}^{N+1} \cap W_{D}^{N+1}=W_{D+E}^{N+1}$ and the corresponding $\sum_{j} c_{j} \ell_{j}$ is precisely multiplication by $-(\tilde{s} / \tilde{t})$, which transforms the space $W_{D+E}^{N+1}$ into the space $W_{2 D}^{N}$.

\section{JACOBIAN ARITHMETIC FOR TYPICAL DIVISOR CLASSES}

In this section, we assemble the results from the previous section, with specific choices of parameters, to give algorithms for typical divisor classes that work for the Jacobian of an arbitrary curve $C$ with a rational point $P_{\infty}$.

Before doing so, we collect here the final statement of our results for the special case of $C_{3,4}$ curves and our previous algorithms:

Theorem 4.1. The algorithms of ASKM07, for addition and doubling in the Jacobian of a $C_{3,4}$ curve work correctly with typical divisors as input, and yield typical 
divisors as output, if and only if the two $\mathbb{K}$-inversions in each algorithm can be carried out.

Proof. All divisors $D$ of degree 3 are represented by elements $F, G$ as in Proposition 2.12, by storing the elements $a, b, \ldots, f$ as well as the inverse $a^{-1}$. Thus $a \neq 0$, and $D$ is typical. Moreover, the algorithm for the "addflip" of two divisors $\left(D, D^{\prime} \mapsto D^{\prime \prime}\right.$ with $\operatorname{div} s=D+D^{\prime}+D^{\prime \prime}$; see below) produces the correct answer, with $D^{\prime \prime}$ typical due to $a^{\prime \prime} \neq 0$ by Propositions 3.3 and 3.10. A similar result holds when we compute the addflip for $D=D^{\prime}$ (called "doubleflip" below), by using Proposition 3.17 instead of Proposition 3.3. The final inversion to obtain the sum or double in the Jacobian (Section 10 of [ASKM07]) is correct by Proposition 3.12

In the appendix, we give formulas for a speedup of the algorithms of ASKM07. by approximately $15 \%$, arising from revisiting the previous work in light of the considerations that led to Proposition 3.18 ,

We now address the generalization to arbitrary $C$. Following the discussion at the beginning of Section 3 , we represent all typical elements of $\operatorname{Pic}^{0}(C)$ as $\left[D-g P_{\infty}\right]$ for a unique typical divisor $D$ of degree $g$. We will occasionally relax this to assume merely that $D$ is semi-typical.

As we have already discussed, "flipping" $D$ corresponds to negation in the Jacobian. The other basic operation in the Jacobian is the "addflip" operation, in the terminology we introduced in KM04. For the rest of this section, it is convenient for us to separate this operation into two cases:

(1) The first case, which we continue to call "addflip", takes as input two typical divisors $D, D^{\prime}$, that one typically hopes are disjoint. Then we want to produce an output divisor $D^{\prime \prime}$ for which there exists a degree $3 g$ element $s \in W_{D+D^{\prime}}^{3 g}$ with divisor div $s=D+D^{\prime}+D^{\prime \prime}$. This means that $\left[D^{\prime \prime}-g P_{\infty}\right]=$ $-\left(\left[D-g P_{\infty}\right]+\left[D^{\prime}-g P_{\infty}\right]\right)$.

(2) The second case, which we call "doubleflip", is the analog of the above in the situation where $D=D^{\prime}$. Thus $\left[D^{\prime \prime}-g P_{\infty}\right]=-2\left[D-g P_{\infty}\right]$ in $\operatorname{Pic}^{0}(C)$, and $s \in W_{2 D}^{3 g}$ satisfies $\operatorname{div} s=2 D+D^{\prime \prime}$.

The basic idea, of course, is that an addflip consists of carrying out an addition, as in Subsection 3.1 (producing a divisor of degree $2 g$ ), followed by a flip as in Subsection 3.2. so the final answer is again a divisor of degree $g$. A doubleflip is similar, except that the initial addition is replaced by a doubling, as in Subsection 3.3. In doing this, we must take care to specify (i) precise values of $N$, and (ii) choices of algorithms to compute the spaces $W_{E}^{N}$ for various intermediate divisors $E$; the goal in doing so is to ensure that we can always certify the final answer after an addflip or a doubleflip to be typical. Alternatively, we can arrange to certify only that a subsequent flip of an addflip or a doubleflip is itself typical, so that the basic operations on typical classes in the Jacobian now become addition and doubling, with which we must also include a certified way of doing negation.

In all the algorithms given below, if the algorithm fails, then it is possible to use the general methods that work for all divisors in KM04, KM07. This will produce a constant slowdown, since the linear algebra involves larger matrices, but is expected to happen so very rarely in typical applications that it can be ignored.

4.1. Direct generalization of the algorithms in [ASKM07. We represent our typical degree $g$ divisor $D$ by a triple $\left(F, G, G_{1}\right)$. Here $D$ is determined by the pair 
$(F, G)$, with $F \in W_{D}^{2 g}, G \in W_{D}^{2 g+1}$, and the "flip" $A$ of $D$ is a degree $g$ divisor, described by the pair $\left(F, G_{1}\right)$. This uses the same $F \in W_{A}^{2 g}$ (because $\operatorname{div} F=$ $D+A$, as usual), and $G_{1} \in W_{A}^{2 g+1}$ is the same element as in Proposition 3.14 We assume that for any input to our algorithms, representing such a divisor $D$ and its complement $A$, we are guaranteed that $D$ and $A$ are both typical.

As written, there is some choice for $F, G$, and $G_{1}$. For example, $D$ determines the pair $(F, G)$ uniquely only up to replacing $(F, G)$ with $\left(a F, a^{\prime} G+b F\right)$, where $a, a^{\prime}, b \in \mathbb{K}$ with $a, a^{\prime} \neq 0$, as observed in Proposition 2.3. In terms of a basis of "monomials" for $\mathcal{R}$, we can make $F$ and $G$ unique by requiring that (i) $F$ and $G$ are monic, and (ii) the coefficient in $G$ of the monomial of degree $2 g$ is zero. As for the choice of $G_{1}$, note that $A$ is uniquely determined by $D$ (or by $\operatorname{div} F$ ), and $G_{1}$ can be modified in the same way as $G$, to yield a unique choice.

Putting together our previous results yields the following theorem.

Theorem 4.2. In the above setting, we have the following algorithms for negation, addition, and doubling of typical divisor classes, with necessary and sufficient conditions under which the algorithms succeed.

(1) Negating a divisor class: Replace the triple $\left(F, G, G_{1}\right)$ by $\left(F, G_{1}, G\right)$. This exchanges the roles of $A$ and $D$, and succeeds in all cases. Both divisors remain typical.

(2) Adding two (different) divisor classes: Briefly, carry out the algorithms in Proposition 3.4 then Proposition 3.8. More specifically, let the two elements of the Jacobian come from divisors $D$ and $D^{\prime}$, represented by the triples $\left(F, G, G_{1}\right)$ and $\left(F^{\prime}, G^{\prime}, G_{1}^{\prime}\right)$. Carry out Step (1) of Proposition 3.4, taking $N=3 g+1$. In other words, compute an echelon basis of the space $W_{D}^{4 g}=F W^{2 g}+G W^{2 g-1}$, and select the first $g+2$ basis elements. This yields a basis for the subspace $W_{D}^{3 g+1}$; similarly, compute $W_{D^{\prime}}^{3 g+1}$. Now compute the intersection to obtain $W_{D+D^{\prime}}^{3 g+1}$, as in the remaining steps of the algorithm in Proposition 3.4. This succeeds if and only if $D$ and $D^{\prime}$ are disjoint, and $D+D^{\prime}$ is semi-typical, in which case we correctly obtain $s \in W_{D+D^{\prime}}^{3 g}, t \in W_{D+D^{\prime}}^{3 g+1}$. Next, apply the flipping algorithm in Proposition [3.8, with $N=2 g+1$. We write as usual $\operatorname{div} s=D+D^{\prime}+D^{\prime \prime}$. The flipping algorithm produces $\left\{F^{\prime \prime}, G^{\prime \prime}\right\}$ that describe $D^{\prime \prime}$; this succeeds if and only if $D+D^{\prime}$ is typical. Do a further flip of $D^{\prime \prime}$ as in Proposition [3.8, again with $N=2 g+1$; assuming this is successful, this produces a divisor $D^{\prime \prime \prime}$, represented by elements $F^{\prime \prime \prime}, G^{\prime \prime \prime}$, and certifies that $D^{\prime \prime}$ is typical. We know that $D^{\prime \prime \prime}$ represents the same class in $\operatorname{Pic}^{0}(C)$ as $D+D^{\prime}$, so $D^{\prime \prime \prime}$ is typical by Corollary [2.6. Thus we return the triple $\left(F^{\prime \prime \prime}, G^{\prime \prime \prime}, G^{\prime \prime}\right)$ (where in fact $\left.F^{\prime \prime \prime}=F^{\prime \prime}\right)$ as our representation of the sum $\left[D^{\prime \prime \prime}-g P_{\infty}\right]=\left[D-g P_{\infty}\right]+\left[D^{\prime}-g P_{\infty}\right]$. Given that $D, D^{\prime}$ and their flips are known to be typical before starting the algorithm, this whole procedure succeeds if and only if $D, D^{\prime}$ are disjoint, and $D^{\prime \prime}$ and $D^{\prime \prime \prime}$ are both typical.

(3) Doubling a divisor class: Start with the usual input data $\left(F, G, G_{1}\right)$, and compute first $H_{1}=-G G_{1} / F$, then $s, t \in W_{2 D}^{3 g+1}$ using Proposition 3.15 with $N=3 g+1$; thus $\operatorname{div} s=2 D+D^{\prime \prime}$. Then do two flips, as in the algorithm for addition in (2) above, thereby obtaining representations of the divisors $D^{\prime \prime}$ and $D^{\prime \prime \prime}$ as in that algorithm. Success occurs if and only if $2 D$ (hence also $D^{\prime \prime \prime}$ ) and $D^{\prime \prime}$ are typical. 
Remark 4.3. Let us bound the probability of failure for the above two algorithms for addition and doubling, when $\mathbb{K}$ is a finite field with $q$ elements, using the results we quoted from KM16, in Theorem 2.10.

For addition to fail, the inputs $D, D^{\prime}$ and output $D^{\prime \prime}$ must satisfy at least one of the following properties:

(1) The divisor $D$, or its flip, is not typical;

(2) The divisor $D^{\prime}$, or its flip, is not typical;

(3) The divisor $D^{\prime \prime}$, or its flip $D^{\prime \prime \prime}$, is not typical;

(4) The divisors $D$ and $D^{\prime}$ are not disjoint.

The probability of at least one of these events happening is at most the sum of their individual probabilities, which we will compute under the uniform distribution on all triples of classes $\left(x, x^{\prime}, x^{\prime \prime}\right)=\left(\left[D-g P_{\infty}\right],\left[D^{\prime}-g P_{\infty}\right],\left[D^{\prime \prime}-g P_{\infty}\right]\right) \in\left(\mathrm{Pic}^{0} C\right)^{3}$ with $x+x^{\prime}+x^{\prime \prime}=0$. Note first that the distribution of each of $x, x^{\prime}$, or $x^{\prime \prime}$ when looked at in isolation is uniform in $\mathrm{Pic}^{0} C$. In fact, any pair made from two of the three entries, such as $\left(x, x^{\prime}\right)$, is uniformly distributed over $\left(\operatorname{Pic}^{0} C\right)^{2}$, because this pair completely determines the third entry (indeed, $x^{\prime \prime}=-x-x^{\prime}$ ). It follows that each of events $(1-3)$ above has probability at most $\left(16^{g} \cdot 2 g+3.4\right) / q$. Moreover, we claim that event $(4)$ has a probability at most $(1.7 \cdot g) / q$. This claim implies that the probability of failure of addition is at most $\left(3 \cdot\left(16^{g} \cdot 2 g+3.4\right)+1.7 \cdot g\right) / q$. For example, if $g=5$, then the numerator does not exceed $3.2 \times 10^{7}$, which means that if $q$ is, say, around $10^{30}$ (around 100 bits), our failure rate is below $3.2 \times 10^{-23}$.

We now explain why our claim holds. For this, it is enough to fix a good $D$ with $\operatorname{deg} D=g$, and bound the probability that $D^{\prime}$ is not disjoint from $D$. Writing $D=E_{1}+\cdots+E_{r}$ as a sum of irreducible divisors with $\sum_{j} \operatorname{deg} E_{j}=g$, the "bad" $D^{\prime}$ are those of the form $E_{j}+E^{\prime}$ with $E^{\prime}$ effective of degree $g-\operatorname{deg} E_{j}$. Let $N_{d}$ denote the number of effective degree $d$ divisors; then the number of bad $D^{\prime}$ is at most $\sum_{j} N_{g-\operatorname{deg} E_{j}}$. The largest this can be is when the $E_{j}$ are all distinct $\mathbb{K}$-rational points, in which case our upper bound for the number of bad $D^{\prime}$ is $g N_{g-1}$, and the probability of the pair $\left(D, D^{\prime}\right)$ not being disjoint is at most $\left(g N_{g-1}\right) /\left|\mathrm{Pic}^{0} C\right|$. On the other hand, we know from equation (2.13) in Proposition 2.15 of [KM16] that $N_{g-1} /\left|\operatorname{Pic}^{0} C\right| \leq 1.7 / q$, and this proves our claim.

A similar argument gives a bound for the probability that doubling will fail. Here the question is how often at least one of $D$ or $2 D$ or their flips can fail to be typical. For uniformly random $D$, this is again at most $\left(16^{g} \cdot 2 g+3.4\right) / q$. However, the class of $2 D$ is not uniform in $\operatorname{Pic}^{0} C$, unless $\left|\operatorname{Pic}^{0} C\right|$ happens to be odd, in which case multiplication by 2 would be a bijection. The worst-case scenario is that the full 2 -torsion of the Jacobian is defined over $\mathbb{K}$, in which case multiplication by 2 is a $2^{2 g}$-to-1 map. In that case, the probability that $2 D$ or its flip is not typical is at most $2^{2 g}\left(16^{g} \cdot 2 g+3.4\right) / q$. (Indeed, if $B \subset \mathrm{Pic}^{0} C$ is the bad set of elements which are either not typical or whose flip is not typical, then the preimage of $B$ under multiplication by 2 cannot have more than $2^{2 g}|B|$ elements.) We deduce that the total probability of failure is at most $\left(2^{2 g}+1\right)\left(16^{g} \cdot 2 g+3.4\right) / q$. For our sample parameters $g=5$ and $q \sim 10^{30}$, this probability is at most $1.1 \times 10^{-20}$.

4.2. A relative of the small model of KM04. We now describe a slight relaxation of the above algorithms, which is close to the "small model" described in Section 5 of KM04. In this setup, we assume only that the divisor $D$ representing 
an element of the Jacobian is semi-typical, but that its flip $\tilde{D}$ is typical; to guarantee correct results, some intermediate results in our computations also need to be certified typical. In this set of algorithms, it occasionally helps to keep track of the penultimate result found, in order to streamline a subsequent calculation.

In this setting, we represent a $D$ of degree $g$ by a triple $\left(W_{D}^{3 g+1}, \tilde{s}, \tilde{t}\right)$. The vector space $W_{D}^{3 g+1}$ is described by an echelon basis, ordered as usual by degree, and we assume that $D$ is semi-typical, and that $D$ is known to be equal to the "flip" of a typical divisor $\tilde{D}$ of degree $\tilde{d}$, with $\tilde{d}=g$ or $2 g$ (we also carry around the value of $\tilde{d})$. The other entries in the triple, $\tilde{s} \in W_{\tilde{D}}^{\tilde{d}+g}$ and $\tilde{t} \in W_{\tilde{D}}^{\tilde{d}+g+1}$, describe $\tilde{D}$ in the usual way; in particular, $\operatorname{div} \tilde{s}=D+\tilde{D}$.

We note here that the vector space $W_{D}^{3 g+1}$ is always base point free; hence this space always determines $D$, regardless of whether $D$ is semi-typical. In any case, having $\tilde{s}$ and $\tilde{t}$ on hand allows us to compute any $W_{D}^{N}$ that we might need, for example if some results end up not being typical and we have to resort to the general-purpose algorithms of [KM07]. We also note that most of the algorithms are valid using the base point free space $W_{D}^{3 g}$, but that this would lead to a longer algorithm for the intersection in the addflip algorithm below. We leave it to the reader to estimate the probability of failure in these algorithms over a large finite field, in the spirit of Remark 4.3.

Theorem 4.4. The following are algorithms for typical divisor classes in the above setting.

(1) Addflip of two divisor classes: Let the classes be represented by the divisors $D$ and $D^{\prime}$. Find the intersection $W_{D}^{3 g+1} \cap W_{D^{\prime}}^{3 g+1}$, as in Steps (2)(4) of Proposition 3.4 with $N=3 g+1$. This succeeds if and only if $D$ and $D^{\prime}$ are disjoint, and $D+D^{\prime}$ is semi-typical. We thus obtain $s, t \in W_{D+D^{\prime}}^{3 g+1}$ with $\operatorname{deg} s=3 g$, deg $t=3 g+1$, and $\operatorname{div} s=D+D^{\prime}+D^{\prime \prime}$. Second, compute $W_{D^{\prime \prime}}^{3 g+1}$ by flipping, as in Proposition 3.8, with $(N, D, A)$ there replaced by $\left(3 g+1, D+D^{\prime}, D^{\prime \prime}\right)$ here. This step succeeds precisely when $D+D^{\prime}$ is typical, and implies that $D^{\prime \prime}$ is semi-typical. We then return the triple $\left(W_{D^{\prime \prime}}^{3 g+1}, s, t\right)$. This whole procedure succeeds if and only if $D, D^{\prime}$ are disjoint, and $D+D^{\prime}$ is typical.

(2) Doubleflip of a divisor class: Given a divisor D representing the class, take the pair $(\tilde{s}, \tilde{t})$, and apply Proposition 3.18 with $N=3 g+1$ to obtain $s, t \in W_{2 D}^{3 g+1}$. Since $\tilde{D}$ is typical, success occurs if and only if $2 D$ is semitypical. Now carry out a flip of $2 D$, using Proposition 3.8 with $N=3 g+1$, to produce the desired answer $W_{D^{\prime \prime}}^{3 g+1}$, and to certify that $2 D$ is typical. If successful, return as before $\left(W_{D^{\prime \prime}}^{3 g+1}, s, t\right)$. This whole procedure succeeds if and only if $2 D$ is typical.

Remark: The algorithm as stated contains some redundancy in the form of repeated computations. Suppose that at the stage prior to starting the doubleflip, we had $\tilde{s}, \tilde{t}$ and were about to carry out a flip to find $W_{D}^{3 g+1}$ to obtain the full triple describing $D$. Then it would be desirable to have some rudimentary lookahead to see whether $D$ will be used as an input for a doubleflip. If so, we can once and for all carry out the algorithm of Proposition 3.18, instead of first ending the previous computation with a simple flip using Proposition 3.8. That way, using Proposition 3.18, we simultaneously obtain both the space $W_{D}^{3 g+1}$ and the elements $s, t \in W_{2 D}^{3 g+1}$. 
(3) Negation of a divisor class: Given the space $W_{D}^{3 g+1}$, take the first two elements $s \in W_{D}^{2 g}, t \in W_{D}^{2 g+1}$ of the echelon basis, with $\operatorname{div} s=D+A$, and flip as in Proposition 3.8 with $N=3 g+1$. This produces the space $W_{A}^{3 g+1}$, while certifying that $D$ was typical to begin with (we already know that $A$ is typical, because $\tilde{D}$ is). Return as output the triple $\left(W_{A}^{3 g+1}, s, t\right)$. This procedure succeeds if and only if $D$ is typical.

4.3. A nontraditional modification. As a last setting in which we can carry out generic algorithms, we describe a change of perspective to the method of Subsection 4.2, Instead of representing the class $\left[D-g P_{\infty}\right] \in \operatorname{Pic}^{0}(C)$ by the triple $\left(W_{D}^{3 g+1}, \tilde{s}, \tilde{t}\right)$, we can view the pair $(\tilde{s}, \tilde{t})$ as itself representing $D$, via the fact that its "opposite" divisor $\tilde{D}$ is determined by $I_{\tilde{D}}=\tilde{s} \mathcal{R}+\tilde{t} \mathcal{R}$. Alternatively, we can recover $D$ from the identity $I_{D}=\{\ell \in \mathcal{R} \mid \ell \tilde{t} \in \tilde{s} \mathcal{R}\}$. This allows us to carry out in essence the same algorithms as in Subsection 4.2, except that now each step begins with our flipping $(\tilde{s}, \tilde{t})$ using either Proposition 3.8 or Proposition 3.18, depending on whether we wish to carry out an addflip or a doubleflip. Then we omit the final flip from the algorithms in the previous subsection. (The same technique works for negation in this model.) Thus we have just shifted our perspective on where the algorithms start and stop, so we do not think of $(\tilde{s}, \tilde{t})$ as being extra baggage that we carry around to speed up some computations, but rather as the actual result. This approach nonetheless comes with two disadvantages. The first, minor, disadvantage, is that a pair $(\tilde{s}, \tilde{t})$ no longer represents a (semi-typical) divisor $D$ uniquely, since there are many choices of $\tilde{D}$ in the same divisor class with $\operatorname{deg} \tilde{D}=2 g$. However, we can always test equality between $(\tilde{s}, \tilde{t})$ and $\left(\tilde{s}^{\prime}, \tilde{t}^{\prime}\right)$ by flipping both and seeing if they yield the same space $W_{D}^{3 g+1}=W_{D^{\prime}}^{3 g+1}$.

The second, more serious, disadvantage is that at the moment when we compute a pair $(\tilde{s}, \tilde{t})$, we have not yet certified that $\tilde{D}$ is typical; this certification happens only after we flip using Proposition 3.8 or Proposition 3.18. Thus in case one of those two algorithms fails, we have no guarantee that $\tilde{s}, \tilde{t}$ are an IGS for $\tilde{D}$, and so we may lose information about what element of the Jacobian we are working with. In that case, we would need to backtrack one full step in the computations to recover the information, and then use a slower general-purpose algorithm.

In conclusion, it is perhaps better in an implementation to stick to the approach of Subsection 4.2, with some lookahead to determine what to do with a particular pair $(\tilde{s}, \tilde{t})$. For purposes of reasoning about the algorithm, however, the point of view in this subsection may be useful.

\section{Appendix: Speedup of the Algorithms for $C_{3,4}$ CURVeS}

In this appendix, we describe a method to combine the computations of Sections 8,9 , and 10 of [ASKM07] into a single more efficient computation. The context here is that we currently know $s, t \in W_{D+D^{\prime}}^{10}$ (where $D$ might equal $D^{\prime}$, and we know that $D+D^{\prime}$ is semi-typical). In our previous algorithms, we did two flips to the pair $\{s, t\}$ to obtain first $D^{\prime \prime}$ and then $D^{\prime \prime \prime}$. By a modification of Propositions 3.14 and 3.18, we can combine these two flips into one computation. We do not know whether these techniques generalize to give a certifiably correct result for other curves, even though they will work generically. In the setting of $C_{3,4}$ curves, however, it is easy to analyze when a divisor is typical, and we can show that the results obtained are correct. 
To start, let us change notation to write $(\tilde{D}, D, A)$ in this appendix, instead of $\left(D+D^{\prime}, D^{\prime \prime}, D^{\prime \prime \prime}\right)$ from ASKM07. Thus we have elements $s \in W_{\tilde{D}}^{9}, t \in W_{\tilde{D}}^{10}$ of the form

$$
\begin{gathered}
s=x^{3}+s_{1} y^{2}+s_{2} x y+s_{3} x^{2}+s_{4} y+s_{5} x+s_{6}, \\
t=x^{2} y+t_{1} y^{2}+t_{2} x y+t_{3} x^{2}+t_{4} y+t_{5} x+t_{6}, \\
\text { with } \operatorname{div} s=\tilde{D}+D, \quad \operatorname{div} t=\tilde{D}+E, \\
\operatorname{deg} \tilde{D}=6, \quad \operatorname{deg} D=3, \text { and } \operatorname{deg} E=4 .
\end{gathered}
$$

Our goal is to find the "flip" $A$ of $D$. Thus $A$ is a divisor with $\operatorname{deg} A=3$, and we wish to compute $F \in W_{A+D}^{6}, G_{0} \in W_{A+E}^{7}$ for which $F t+G_{0} s=0$. We want to do so while certifying in the process that $\{s, t\}$ is an IGS for $\tilde{D}$ (i.e., $D$ and $E$ are disjoint) and that $\tilde{D}$ is typical, whence so is $A$. (The reason for writing $G_{0}$ is that the final $G \in W_{A}^{7}$ will be a slight modification.) As usual, we will compute with the apparently weaker system of equations $F t+$ $G_{0} s \equiv 0 \bmod W^{8}$. This amounts to finding a linear combination of $t, x t, y t, x^{2} t$ and $s, x s, y s, x^{2} s, x y s$ that vanishes when viewed in the quotient space $W^{16} / W^{8}$. We represent elements of this quotient space as column vectors with respect to the basis $\left\{x^{3}, x^{2} y, x y^{2}, y^{3}, x^{3} y, x^{2} y^{2}, x y^{3}, y^{4}\right\}$, analogously to equation (17) of ASKM07. Adapting the entries of the matrix $N$ in Lemma 8.1 of that article, we have that our desired images of $t, x t, y t, x^{2} t, s, x s, y s, x^{2} s, x y s$ are the columns $C_{1}, \ldots, C_{9}$ of the matrix

$$
\left(\begin{array}{ccccccccc}
0 & t_{3} & 0 & t_{5} & 1 & s_{3} & 0 & s_{5}+q_{2} & 0 \\
1 & t_{2} & t_{3} & t_{4}+q_{2}+t_{3} p_{2} & 0 & s_{2}+p_{2} & s_{3} & s_{4}+p_{1}+s_{3} p_{2} & s_{5}+q_{2} \\
0 & t_{1} & t_{2} & p_{1} & 0 & s_{1} & s_{2} & 0 & s_{4}+p_{1} \\
0 & 0 & t_{1} & t_{3} & 0 & 1 & s_{1} & s_{3} & 0 \\
0 & 1 & 0 & t_{2} & 0 & 0 & 1 & s_{2}+p_{2} & s_{3} \\
0 & 0 & 1 & t_{1}+p_{2} & 0 & 0 & 0 & s_{1} & s_{2}+p_{2} \\
0 & 0 & 0 & 0 & 0 & 0 & 0 & 1 & s_{1} \\
0 & 0 & 0 & 1 & 0 & 0 & 0 & 0 & 1
\end{array}\right)
$$

Note that these columns are different from those in Section 9 of ASKM07, where we had 11 columns representing elements of $W^{17} / W^{9}$. The $p_{i}$ and $q_{i}$ are constants arising from the equation of the curve $C$. We emphasize that we do not compute the above matrix directly, since this would involve the two products $t_{3} p_{2}$ and $s_{3} p_{2}$, which we do not need separately, but can fold into other parts of the computation.

As in our earlier article, we count the complexity of a computation in terms of the number of multiplications $M$ and inversions $I$ it takes in the field $\mathbb{K}$. We ignore additions and subtractions, as well as multiplications and divisions by 2 in $\mathbb{K}$; recall that we assume in [ASKM07] that $\mathbb{K}$ does not have characteristic 2 or 3.

The first stage of the computation is to compute three quantities $\ell_{1}, \ell_{2}, \ell_{3}$ that will be useful later, for which nonvanishing of $\ell_{1}$ is equivalent to $\tilde{D}$ (and hence $A$ ) being typical:

Lemma A.1. Using $3 M$, we can compute

$$
\ell_{1}=t_{1}-s_{2}+s_{1}^{2}, \quad \ell_{2}=t_{2}-s_{3}+s_{1}\left(s_{2}+p_{2}\right), \quad \ell_{3}=t_{3}+s_{1} s_{3} .
$$

We then have: 
(1) The combination of columns $C_{2}^{\prime}=C_{2}-C_{7}+s_{1} C_{6}$, which represents $x t-$ $y s+s_{1} x s$, is equal to the column vector $\left(\ell_{3}, \ell_{2}, \ell_{1}, 0,0,0,0,0\right)^{\mathbf{T}}$;

(2) Similarly, $C_{4}^{\prime}=C_{4}-C_{9}+s_{1} C_{8}$, which represents $x\left(x t-y s+s_{1} x s\right)$, has the form $\left(*, *, *, \ell_{3}, \ell_{2}, \ell_{1}, 0,0\right)^{\mathbf{T}}$;

(3) The divisor $\tilde{D}$ is typical if and only if $\ell_{1} \neq 0$.

Proof. Statements (1) and (2) are direct computations. Only statement (3), about typicality, needs proof. Now $\tilde{D}$ is typical if and only if we have invertibility of the $7 \times 7$ submatrix of (A.2) obtained from the columns corresponding to $\left\{t, x t, y t, s, x s, y s, x^{2} s\right\}$ and the first seven rows, since this corresponds to having $s W^{6}+t W^{5}+W^{8}=W^{15}$. The columns in question are all except $C_{4}$ and $C_{9}$, and we can further replace $C_{2}$ by $C_{2}^{\prime}$, as given above, without affecting the invertibility; but in that case the columns can be rearranged to form a triangular matrix with diagonal entries all 1 , except for a single $\ell_{1}$. This proves our result.

We now define four more quantities $m_{0}, m_{1}, m_{2}, m_{3}$ by:

$$
\begin{aligned}
& m_{0}=\ell_{3}-\ell_{1} t_{1}, \\
& m_{1}=-s_{4}-\left(\ell_{1} t_{2}+\ell_{2} t_{1}\right)-m_{0} s_{1}, \\
& m_{2}=t_{4}-s_{5}+s_{1}\left(s_{4}+p_{1}\right)+p_{2} \ell_{3}-\left(\ell_{1} t_{3}+\ell_{2} t_{2}\right)-m_{0}\left(s_{2}+p_{2}\right), \\
& m_{3}=t_{5}+s_{1}\left(s_{5}+q_{2}\right)-\ell_{2} t_{3}-m_{0} s_{3} .
\end{aligned}
$$

The motivation for the above quantities is that $C_{4}^{\prime \prime}=C_{4}^{\prime}-\ell_{1} C_{3}-\ell_{2} C_{2}$ has the form $\left(*, *, *, m_{0}, 0,0,0,0\right)^{\mathbf{T}}$, while $C_{4}^{\prime \prime \prime}=C_{4}^{\prime \prime}-m_{0} C_{6}=\left(m_{3}, m_{2}, m_{1}, 0,0,0,0,0\right)^{\mathbf{T}}$. However, this fact is not needed to verify our proof below.

Lemma A.2. One can compute $m_{0}, \ldots, m_{3}$ using only $10 M$, as opposed to the $12 M$ apparent in (A.4).

Proof. The point is that the four expressions $\alpha=\ell_{1} t_{1}, \beta=\ell_{1} t_{2}+\ell_{2} t_{1}, \gamma=\ell_{1} t_{3}+$ $\ell_{2} t_{2}, \delta=\ell_{2} t_{3}$ can be computed using just $4 M$ instead of the apparent $6 M$. This is equivalent to Toom-Cook multiplication of polynomials via interpolation at 0,1 , -1 , and " $\infty$ ". Explicitly, use $4 M$ to compute $t_{1} \ell_{1}, \ell_{2} t_{3},\left(t_{1}+t_{2}+t_{3}\right)\left(\ell_{1}+\ell_{2}\right)$, and $\left(t_{1}-t_{2}+t_{3}\right)\left(\ell_{1}-\ell_{2}\right)$. Thus we know the quantities $\alpha, \delta, \alpha+\beta+\gamma+\delta, \alpha-\beta+\gamma-\delta$. Hence we also know $\beta \pm \gamma$ at no extra cost (of multiplications $M$ ), and can determine $\beta, \gamma$ at no further cost, because division by 2 is also "free" in our model.

Proposition A.3. Given $\ell_{1}, \ell_{2}, \ell_{3}, m_{0}, m_{1}, m_{2}, m_{3}$ as above, one can at a further cost of $1 I, 4 M$ compute $\ell_{1}^{-1}, m_{1} / \ell_{1},\left(m_{1} / \ell_{1}\right) \ell_{2},\left(m_{1} / \ell_{1}\right) \ell_{3},\left(m_{1} / \ell_{1}\right) s_{1}$, thereby obtaining the following values of $F, G_{0}$ :

$$
\begin{aligned}
F & =x^{2}-\ell_{1} y-\left(\frac{m_{1}}{\ell_{1}}+\ell_{2}\right) x+\left(\frac{m_{1}}{\ell_{1}}\right) \ell_{2}-m_{2}, \\
G_{0} & =-x y+s_{1} x^{2}+\left(\frac{m_{1}}{\ell_{1}}\right) y-\left(m_{0}+\left(\frac{m_{1}}{\ell_{1}}\right) s_{1}\right) x+\left(\frac{m_{1}}{\ell_{1}}\right) \ell_{3}-m_{3} .
\end{aligned}
$$

Being able to invert $\ell_{1}$ certifies that $\tilde{D}$ is typical, and that the above computation correctly finds $F \in W_{D}^{6}$. Writing $\operatorname{div} F=D+A$, we also obtain that $\operatorname{div} G_{0}=A+E$, and that the pair $(F, G)$ with $G=-G_{0}+s_{1} F$ is an IGS for the typical divisor $A$. It costs a further $3 M$ to compute the coefficients of $G$ from $F$ and $G_{0}$. Thus the total cost of this proposition is $1 I, 7 M$, if done in two stages. However, it is possible to bring the total cost down to $1 I, 6 \mathrm{M}$, by combining both parts of the computation to yield $F$ and $G$ directly. 
Proof. One can check by a lengthy calculation (preferably using a computer) that $F t+G_{0} s \equiv 0 \bmod W^{8}$; this amounts to checking that the appropriate linear combination of columns of (A.2) vanishes. We have already shown that invertibility of $\ell_{1}$ implies that $\tilde{D}$ is typical. This implies that the divisors $D$ and $E$ from (A.1) are disjoint, and that $W_{\tilde{D}}^{8}=0$, so we obtain as usual that $F t+G_{0} s=0$, and that $F \in W_{D}^{6}$. The statement about $\operatorname{div} G_{0}$ follows. Computing $G$ from $G_{0}$ involves $3 M$ because we need to multiply $s_{1}$ by each of the coefficients $\ell_{1},\left(\frac{m_{1}}{\ell_{1}}+\ell_{2}\right),\left(\left(\frac{m_{1}}{\ell_{1}}\right) \ell_{2}-m_{2}\right)$ of $F$. We thus obtain a pair $(F, G)$ in $W_{A}^{7}$ whose $F$ has a coefficient $-\ell_{1}$ for the $y$ monomial. Thus we have obtained a description of the divisor $A$ as in ASKM07, with the equivalent of $a \neq 0$ from Proposition 2.12 and no added cost to compute $a^{-1}=-\ell_{1}^{-1}$.

We now explain the extra saving of $1 M$ from folding the computations together. This comes from the coefficient of $x$ in $G$. As stated currently, it appears to take $2 M$ to compute this coefficient: (i) the first $M$ comes from the multiplication $\left(m_{1} / \ell_{1}\right) \cdot s_{1}$, to compute the coefficient of $x$ in $G_{0}$, which is $-\left(m_{0}+\left(m_{1} / \ell_{1}\right) s_{1}\right)$; (ii) the second $M$ comes when we compute $G=-G_{0}+s_{1} F$, since we multiply $s_{1}$ by the coefficient of $x$ in $F$, which is $-\left(\left(m_{1} / \ell_{1}\right)+\ell_{2}\right)$. However it is immediate that the coefficient of $x$ in $G$ that results from this is

$$
-\left(-\left(m_{0}+\left(m_{1} / \ell_{1}\right) s_{1}\right)\right)+s_{1}\left(-\left(\left(m_{1} / \ell_{1}\right)+\ell_{2}\right)\right)=m_{0}-s_{1} \ell_{2},
$$

which can naturally be computed using the single $M$ of $s_{1} \cdot \ell_{2}$. This concludes the proof.

Combining Lemmas A.1 and A.2 with Proposition A.3, we obtain the following result:

Theorem A.4. The above procedure produces the same effect as Proposition 9.3 and Proposition 10.1(i) of [ASKM07. This means that we can use a total of $19 M, 1 I$ to replace what took us $38 M, 1 I$ in ASKM07. Consequently, the cost of Jacobian operations in a $C_{3,4}$ curve can be reduced by $19 M$ to obtain that addition of typical elements can be carried out using $98 M, 2 I$ while doubling can be carried out using $110 M, 2 I$. The results are certified to be correct and typical, provided all inverses can be computed. This represents a further speedup of approximately 15\% over the results of that article.

\section{REFERENCES}

[ASKM07] Fatima K. Abu Salem and Kamal Khuri-Makdisi, Fast Jacobian group operations for $C_{3,4}$ curves over a large finite field, LMS J. Comput. Math. 10 (2007), 307-328. MR 2335723 (2008d:14096)

[BEFG04] Abdolali Basiri, Andreas Enge, Jean-Charles Faugère, and Nicolas Gürel, Implementing the arithmetic of $C_{3,4}$ curves, Algorithmic number theory-ANTS VI, Lecture Notes in Comput. Sci., vol. 3076, Springer, Berlin, 2004, pp. 87-101. MR 2137346 (2006a:14101)

[BEFG05] - The arithmetic of Jacobian groups of superelliptic cubics, Math. Comp. 74 (2005), no. 249, 389-410 (electronic). MR 2085899 (2005f:11126)

[FO04] Stéphane Flon and Roger Oyono, Fast arithmetic on Jacobians of Picard curves, Public key cryptography - PKC 2004, Lecture Notes in Comput. Sci., vol. 2947, Springer, Berlin, 2004, pp. 55-68. MR 2095638 (2005k:14049)

[FOR08] Stéphane Flon, Roger Oyono, and Christophe Ritzenthaler, Fast addition on nonhyperelliptic genus 3 curves, Algebraic geometry and its applications, Ser. Number Theory Appl., vol. 5, World Sci. Publ., Hackensack, NJ, 2008, pp. 1-28. MR 2484046 (2009m:14044) 
ON JACOBIAN GROUP ARITHMETIC FOR TYPICAL DIVISORS ON CURVES

[KM04] Kamal Khuri-Makdisi, Linear algebra algorithms for divisors on an algebraic curve, Math. Comp. 73 (2004), no. 245, 333-357 (electronic). MR 2034126 (2005a:14081)

[KM07] Asymptotically fast group operations on Jacobians of general curves, Math. Comp. 76 (2007), no. 260, 2213-2239 (electronic). MR 2336292 (2009a:14072)

[KM16] Upper bounds for some Brill-Noether loci over a finite field, available from http://arxiv.org/abs/1609.03349, DOI 10.1142/S1793042118500471, to appear in International Journal of Number Theory, preprint, 2016.

[OT13] Roger Oyono and Nicolas Thériault, Group arithmetic in $C_{3,5}$ curves, J. Symbolic Comput. 56 (2013), 1-26. MR 3061706

Mathematics Department, American University of Beirut, Bliss Street, Beirut, Lebanon

E-mail address: kmakdisi@aub.edu.lb 